\author{
Режимы динамики популяции \\ с неперекрывающимися поколениями \\ с учетом генетической и стадийной структур \\ Г. П. Неверова ${ }^{1, a}$, Е. Я. Фрисман ${ }^{2, b}$ \\ ${ }^{1}$ Институт автоматики и процессов управления ДВО РАН, \\ Россия, 690041, г. Владивосток, ул. Радио, д. 5 \\ ${ }^{2}$ Институт комплексного анализа региональных проблем ДВО РАН, \\ Россия, 679016, г. Биробиджан, ул. Шолом-Алейхема, д. 4 \\ E-mail: ${ }^{a}$ galina.nev@gmail.com, ${ }^{b}$ frisman@mail.ru
}

Получено 14.05.2020, после доработки - 17.06.2020.

Принято к публикации 29.06.2020.

В данной работе рассматривается простейшая модель динамики популяции с неперекрывающимися поколениями, в которой плотностно-зависимые факторы лимитируют интенсивность рождаемости. При этом репродуктивный потенциал определяется генетически, а процессы размножения приурочены к определенному годовому сезону. Исследуемая в работе эколого-генетическая модель представляет собой объединение экологической модели динамики лимитированной популяции с неперекрывающимися поколениями и микроэволюционной модели динамики ее генетической структуры для случая, когда адаптивное разнообразие репродуктивных возможностей в популяции определяется одним аутосомным диаллельным локусом с аллеломорфами $A$ и $a$. В ходе исследования данной модели показано, что генетический состав популяции (а именно, будет ли она полиморфной или мономорфной) определяется значениями репродуктивных потенциалов гетерозиготы и гомозигот. При этом режимы динамики численности популяции определяются величиной среднего репродуктивного потенциала зрелых особей и интенсивностью процессов саморегуляции. В частности, показано, что эволюционный рост среднего значения репродуктивного потенциала при плотностной регуляции рождаемости приводит к дестабилизации динамики численности возрастных групп. В то время как интенсивность процессов саморегуляции определяет характер возникающих колебаний, поскольку от количественной оценки именно этого фактора зависит сценарий потери устойчивости равновесных состояний. Показано, что закономерности возникновения и эволюции циклических режимов динамики в большой степени определяются особенностями жизненного цикла особей, составляющих популяцию. Именно жизненный цикл определяет наличие изолированных субпопуляций разных лет, что, в свою очередь, приводит к возможности независимой микроэволюции этих субпопуляций и возникновения сложных сценариев динамики как численности, так и генетической структуры. Закрепление разных адаптивных мутаций постепенно приведет к генетической (а возможно, и морфологической) дифференциации и к различиям в средних репродуктивных потенциалах субпопуляций и достижению ими разного равновесного уровня численности. Дальнейший эволюционный рост репродуктивных потенциалов экологически лимитированных субпопуляций приводит к колебаниям их численности, которые могут отличаться не только амплитудой, но и фазой. Обнаруженные в предложенной модели сценарии микроэволюции генетического состава популяции, связанные с колебаниями численности, вполне согласуются с результатами исследований популяции тихоокеанской горбуши, которая демонстрирует не только колебания численности, но и наличие генетически дифференцированных субпопуляций смежных поколений.

Ключевые слова: популяционная динамика, стадийная и генетическая структуры, плотностно-зависимая регуляция, динамические режимы, мультистабильность, генетическое разнообразие, смена режима динамики, смена направления эволюции

Работа выполнена в рамках государственных задании Института автоматики и процессов управления ДВО РАН, Института комплексного анализа региональных проблем ДВО РАН.

(C) 2020 Галина Петровна Неверова, Ефим Яковлевич Фрисман Статья доступна по лицензии Creative Commons Attribution-NoDerivs 3.0 Unported License. Чтобы получить текст лицензии, посетите веб-сайт http://creativecommons.org/licenses/by-nd/3.0/ или отправьте письмо в Creative Commons, PO Box 1866, Mountain View, CA 94042, USA. 
UDC: $51-76: 574.34$

\title{
Dynamics regimes of population with non-overlapping generations taking into account genetic and stage structures
}

\author{
G. P. Neverova ${ }^{1, a}$, E. Ya. Frisman ${ }^{2, b}$ \\ ${ }^{1}$ Institute of Automation and Control Processes, Far Eastern Branch of RAS, \\ 5 Radio st., Vladivostok, 690041, Russia \\ ${ }^{2}$ Institute for Complex Analysis of Regional Problems, Far Eastern Branch of RAS, \\ 4 Sholom-Aleikhem st., Birobidzhan, 679016, Russia

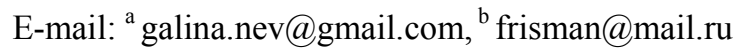

Received 14.05.2020, after completion - 17.06.2020.

Accepted for publication 29.06.2020.

This paper studies a model of a population with non-overlapping generations and density-dependent regulation of birth rate. The population breeds seasonally, and its reproductive potential is determined genetically. The model proposed combines an ecological dynamic model of a limited population with non-overlapping generations and microevolutionary model of its genetic structure dynamics for the case when adaptive trait of birth rate controlled by a single diallelic autosomal locus with allelomorphs A and a. The study showed the genetic composition of the population, namely, will it be polymorphic or monomorphic, is mainly determined by the values of the reproductive potentials of heterozygote and homozygotes. Moreover, the average reproductive potential of mature individuals and intensity of self-regulation processes determine population dynamics. In particularly, increasing the average value of the reproductive potential leads to destabilization of the dynamics of age group sizes. The intensity of self-regulation processes determines the nature of emerging oscillations, since scenario of stability loss of fixed points depends on the values of this parameter. It is shown that patterns of occurrence and evolution of cyclic dynamics regimes are mainly determined by the features of life cycle of individuals in population. The life cycle leading to existence of non-overlapping generation gives isolated subpopulations in different years, which results in the possibility of independent microevolution of these subpopulations and, as a result, the complex dynamics emergence of both stage structure and genetic one. Fixing various adaptive mutations will gradually lead to genetic (and possibly morphological) differentiation and to differences in the average reproductive potentials of subpopulations that give different values of equilibrium subpopulation sizes. Further evolutionary growth of reproductive potentials of limited subpopulations leads to their number fluctuations which can differ in both amplitude and phase.

Keywords: population dynamics, stage and genetic structures, density-dependent regulation, dynamic modes, multistability, genetic diversity, shifting dynamic modes, change in direction of evolution

Citation: Computer Research and Modeling, 2020, vol. 12, no. 5, pp. 1165-1190 (Russian).

The work was performed within the scope of the state tasks of the Institute of Automation and Control Processes FEB RAS, and the Institute for Complex Analysis of Regional Problems FEB RAS. 


\section{Введение}

Возникновение и исчезновение колебаний в динамике численности популяций, а также механизмы и причины их вызывающие, являются одной из самых обсуждаемых тем в экологии [Гиммельфар и др., 1974; Дажо, 1975; Одум, 1975; Уильямсон, 1975; Свирежев, Логофет, 1978; Gurney, Nisbet, 1998; Inchausti, Ginzburg, 1998; Ginzburg, Colyvan, 2004; Barraquand et al., 2017]. Ведущей концепцией о причинах колебаний является теория саморегуляции [Lack, 1954; Гиммельфар и др., 1974; Дажо, 1975; Одум, 1975; Уильямсон, 1975; Свирежев, Логофет, 1978; Boer, Reddingius, 1996; Gurney, Nisbet, 1998; Inchausti, Ginzburg, 1998; Ginzburg, Colyvan, 2004; Barraquand et al., 2017], в которой утверждается, что в силу переуплотнения происходит снижение либо выживаемости, либо рождаемости. На наш взгляд, действительно механизмы плотностной регуляции являются тем фундаментом, на базе которого формируются периодические колебания численности. Так, при низкой численности, когда хватает ресурсов, будет наблюдаться рост численности популяции, причем в несколько этапов с замедлением скорости роста, поскольку ресурсов становится меньше. Затем происходит падение численности популяции в силу переуплотнения и, следовательно, возросшей конкуренции за ресурсы, приводящей к запуску процессов саморегуляции, ведущих к снижению либо рождаемости [Дажо, 1975; Одум, 1975; Уильямсон, 1975; Чернявский, Лазуткин, 2004; Фрисман, Ласт, Лазуткин, 2010; Новиков, Панов, Мошкин, 2012; Krebs, 2013], либо выживаемости [Дажо, 1975; Одум, 1975; Уильямсон, 1975].

Однако для описания динамики реальных популяций учитывать только плотностнозависимую регуляцию недостаточно, поскольку существуют и другие важные факторы, оказывающие влияние на появление колебаний, например сезонность размножения. Действительно, наиболее яркие примеры колебательных режимов динамики численности демонстрируют виды с неперекрывающимися поколениями и коротким жизненным циклом, при этом процесс размножения у таких видов приурочен к конкретному времени года [Lack, 1954; Ricker, 1954; Дажо, 1975; Одум, 1975]. Как оказалось, использование дискретных во времени моделей, учитывающих сезонность размножения, позволяет получить колебания даже при размерности системы, равной единице; при этом траектории модели имеют «пилообразный характер», который возникает в результате потери устойчивости нетривиального равновесия через каскад бифуркаций удвоения периода [Ricker, 1954; Мау, 1974; Шапиро, Луппов, 1983].

Еще одним важным фактором, который необходимо учитывать, является стадийная структура популяции, поскольку особи разного возраста характеризуются разной выживаемостью и разной степенью участия в размножении. Как оказалось, учет стадийной или возрастной структуры расширяет диапазон динамического поведения систем, учитывающих сезонность размножения, поскольку возникает еще один сценарий потери устойчивости - сценарий Неймарка-Сакера [Шапиро, 1983; Шапиро, Луппов, 1983; Фрисман, 1994; Frisman, Neverova, Revutskaya, 2011; Жданова, Фрисман, 2011, Фрисман и др., 2019]. Бифуркация Неймарка-Сакера в дискретных во времени моделях является аналогом бифуркации Андронова-Хопфа в непрерывных и приводит к рождению инвариантной кривой. Как результат, дискретные во времени модели с учетом возрастной структуры способны описывать не только длиннопериодические колебания, которые наблюдаются в моделях с непрерывным временем, но и «зашумленные колебания». Данное разнообразие динамических режимов возможно за счет разных квазипериодических режимов, вид которых определяется порядком обхода предельной инвариантной кривой [Кузнецов и др., 2012а]. В частности, если точки фазовой траектории последовательно заполняют фазовый портрет, представляющий собой инвариантную кривую, то наблюдаются длиннопериодические колебания, подобные поведению траекторий в непрерывных моделях. В случае же «зашумленного цикла» инвариантная кривая заполняется в соответствии с движением по элементам цикла, при этом наблюдается не точное попадание в элементы цикла, а некоторое смещение относительно каждого элемента, что и приводит к «размыванию» цикла.

Резюмируя все вышенаписанное, можно заключить, что модель динамики численности популяции с неперекрывающимися поколениями и коротким жизненным циклом должна учитывать сезонность размножения, плотностно-зависимую регуляцию и возрастную структуру 
популяции. Однако хотелось бы заострить внимание на еще одном факте, обнаруженном в ходе наблюдений и лабораторных исследований, который может оказывать влияние на развитие таких популяций. Как оказалось, в случае изоляции между поколениями, которая возможна за счет гибели зрелых особей после процесса размножения, наблюдается выраженная генетическая дифференциация особей разных поколений. Примером таких популяций является популяция тихоокеанской горбуши (Oncorhynchus gorbuscha), вида, нерестящегося на втором году жизни [Животовский и др., 1989; Ефанов, 2005; Пустовойт, 2011, 2017; Beacham et al., 2012; Sato, Urawa, 2017]. Благодаря этой особенности в одной реке размножаются особи четных и нечетных генераций, между которыми существует жесткая репродуктивная изоляция. В результате две субпопуляции четных и нечетных лет порождают двухлетний цикл. На основе данных об аллозимной изменчивости было показано наличие заметных генетических различий между поколениями четных и нечетных лет, которые демонстрируют генетическую дифференциацию [Животовский и др., 1989; Zhivotovsky et al., 1994; Ефремов, 2002; Пустовойт, 2011, 2017; Beacham et al., 2012; Sato, Urawa, 2017]. Как результат, каждое поколение характеризуется своим набором и частотой встречаемости генов, что сказывается на демографических параметрах и, соответственно, развитии популяции в целом. Таким образом, дополнительно при моделировании динамики популяций с неперекрывающимися поколениями необходимо учитывать генетическую структуру популяции.

В данной работе рассматривается простейшая модель популяции с неперекрывающимися поколениями, в которой плотностно-зависимые факторы лимитируют интенсивность рождаемости, но при этом репродуктивный потенциал определяется генетически, а процессы размножения приурочены к определенному годовому сезону. Основное внимание уделяется возникающим динамическим режимам и нелинейным динамическим эффектам. Исследуемая в работе эколого-генетическая модель представляет собой объединение экологической модели динамики лимитированной популяции с неперекрывающимися поколениями и микроэволюционной модели динамики ее генетической структуры.

\section{Модель динамики численности лимитированной популяции с неперекрывающимися поколениями}

Как уже указывалось, наиболее ярким примером вида с неперекрывающимися поколениями и выраженной стадийностью развития является тихоокеанская горбуша (Oncorhynchus gorbuscha). Особи этого проходного вида созревают на втором году жизни и идут на нерест летом или ранней осенью из морской воды в пресноводный бассейн, где откладывают икру и гибнут после нереста. Вылупившиеся из икры мальки зимуют в пресноводной среде, а весной скатываются в море и там переживают лето, осень, зиму и следующую весну, вплоть до полного созревания. Таким образом, к моменту начала нереста каждая локальная популяция горбуши оказывается представлена двумя субпопуляциями разных стадий развития: 2-летними половозрелыми особями, идущими на нерест, и неполовозрелыми годовалыми особями, продолжающими нагул в море.

Построим модель динамики численности такой популяции. Пусть $X_{n}-$ численность годовалых особей, а $Y_{n}$ - численность половозрелых особей в $n$-м периоде размножения (точнее, к началу периода размножения в $n$-м году). Между $n$-м и $(n+1)$-м периодами размножения произойдут следующие события: взрослые особи отнерестятся и погибнут, выжившая отложенная оплодотворенная икра разовьется до мальков, а выжившие мальки скатятся в море и подрастут до годовиков. Вместе с тем за то же время выжившие годовики разовьются до половозрелого возраста и пойдут на нерест. Таким образом, к началу следующего $(n+1)$-го периода размножения окажется, что

$$
\begin{aligned}
& X_{n+1}=Q_{n} \cdot w \cdot v \cdot Y_{n}, \\
& Y_{n+1}=s X_{n},
\end{aligned}
$$


где $Q_{n}$ - среднее количество икры, откладываемое одной половозрелой самкой, $w-$ доля самок в популяции, $v$ - выживаемость икры и мальков на первом году жизни, $s-$ выживаемость особей на втором году жизни.

Количество икры зависит от упитанности особей. Упитанность в большой степени определяется запасом кормовых ресурсов. Чем больше запасов приходится на одну особь, тем больше упитанность. В море эти ресурсы распределяются между особями разных возрастных стадий. Чем больше численность каждого из возрастных классов, тем меньше ресурсов приходится на одну особь. Падение упитанности (и, соответственно, количества икры) с ростом численности возрастных классов будем описывать экспоненциальной функцией следующего вида:

$$
Q_{n}=Q \cdot \exp \left(-\alpha X_{n}-\beta Y_{n}\right),
$$

где $Q$ - максимально возможное количество икры у самки горбуши, а $\alpha$ и $\beta$ - коэффициенты, характеризующие степени воздействия численностей особей ювенильной и зрелой групп на уменьшение упитанности (определяемыми их интенсивностями потребления ресурсов и особенностями нагула). Эта зависимость выбрана по аналогии с функцией «запас-потребление», предложенной У. Рикером именно для популяций лососевых видов рыб [Ricker, 1954]. Фактически (2) описывает плотностно-зависимую регуляцию уровня воспроизводства популяции горбуши.

Подставляя (2) в (1), получаем искомые уравнения динамики численности локальной популяции биологического вида, отдельные поколения которого не перекрываются, но особи проходят разные стадии развития и осуществляется плотностно-зависимая регуляция процесса воспроизводства:

$$
\begin{aligned}
& X_{n+1}=B \cdot Y_{n} \exp \left(-\alpha X_{n}-\beta Y_{n}\right), \\
& Y_{n+1}=s X_{n},
\end{aligned}
$$

где $B=Q \cdot w \cdot v$ - репродуктивный потенциал половозрелых особей с учетом выживаемости потомков до годовалого возраста.

Переход к безразмерным переменным - относительным численностям $s \beta X \rightarrow x$ и $\beta Y \rightarrow y-$ позволяет «избавиться» от двух параметров и свести модель (3) к виду

$$
\left\{\begin{array}{l}
x_{n+1}=r y_{n} \exp \left(-\rho x_{n}-y_{n}\right), \\
y_{n+1}=x_{n},
\end{array}\right.
$$

где $\rho=\alpha /(s \beta), r=s B$. Коэффициент $r$ характеризует репродуктивный потенциал старшего возрастного класса с учетом выживаемости потомков до взрослого возрастного класса, параметр $\rho$ - относительный вклад младшей возрастной группы в лимитирование процесса воспроизводства.

Модель (4) имеет две стационарные точки: тривиальную и нетривиальную. Область устойчивости нетривиального решения представлена на рис. 1 , $a$, ее границы найдены на основе стандартной методики [Шапиро, Луппов, 1983; Кузнецов и др., 2012а]. Как видно, при $\rho<1$ потеря устойчивости происходит по сценарию Неймарка-Сакера (бифуркационная линия $\left.\lambda=e^{ \pm i \theta}\right)$, при $\rho=1$ в точке $\left(r=e^{4}, \rho=1\right)$ наблюдается простейшая бифуркация коразмерности два: резонанс $1: 2$, также при $\rho=1$ реализуется переход через -1 (бифуркационная линия $\lambda=-1$ ). Эволюция динамических режимов при изменении значений параметров в области неустойчивости представлена на карте динамических режимов (рис. 1, б). Как видно, переход через линию $\lambda=e^{ \pm i \theta}$ сопровождается возникновением характерной для бифуркации Неймарка-Сакера картины, которая состоит в возникновении области квазипериодических режимов $Q$ с погруженной в нее системой языков Арнольда (рис. 1, б). Особого внимания заслуживает переход собственных чисел системы (4) через -1 , т. е. прямую $\rho=1$. Как видно на карте, во всей области $\rho>1$ наблюдается каскад бифуркаций удвоения периода, который происходит с ростом значений параметра $r$ и запускается при переходе через $r=1$, а не при переходе собственных чисел характеристического многочлена через -1 , т. е. прямую $\rho=1$. Соответственно, граница $\rho=1$ от- 
вечает не удвоению периода, а жесткому переходу через мультипликатор -1 [Кузнецов и др., 2012a, 2012б].

Также можно наблюдать область 3-цикла, которая перекрывает область устойчивости нетривиального решения системы (4). 3-цикл устойчив одновременно с равновесным состоянием. Как показано в наших предыдущих работах, 3-цикл является результатом касательной бифуркации, т. е. в модели возникает мультистабильность. И, следовательно, в этой области начальное условие оказывает влияние на тип достигаемого динамического режима. В частности, на рис. 1, в представлен бассейн притяжения динамических режимов модели (4) из области мультистабильности. Как видно, при одних начальных условиях система стабилизируется, а при других демонстрирует трехгодичные колебания (рис. 1, в).

(a)

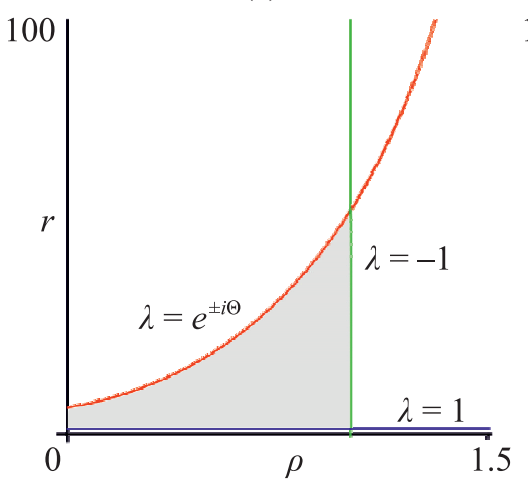

(б) $x_{0}=y_{0}=0.1$

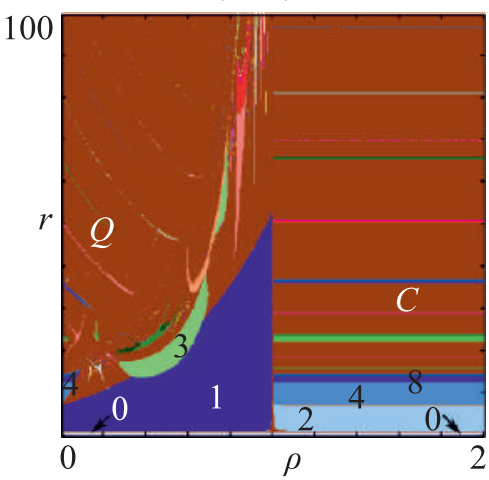

(в) $r=16, \rho=0.5$

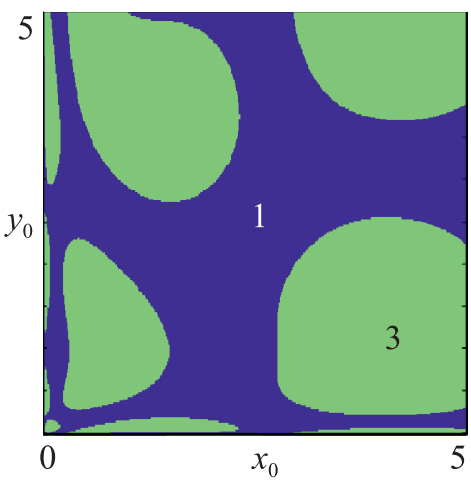

Рис. 1. а) Область устойчивости нетривиального решения системы (4). б) Карта динамических режимов системы (4). Числа соответствуют длине наблюдаемого цикла, $Q$ - квазипериодическая динамика, $C$ хаотическая динамика, 0 - вырождение популяции. в) Бассейны притяжения сосуществующих динамических режимов в области мультистабильности

\section{Введение в модель микроэволюционных процессов, влияющих на интенсивность воспроизводства}

Предположим теперь, что рождаемость меняется в процессе микроэволюции, т. е. репродуктивный потенциал - максимально возможная плодовитость особей - является адаптивным признаком, который определяется генетически. Для понимания основных закономерностей эволюции динамического поведения численности лимитированной популяции ограничимся подробным рассмотрением простой модельной ситуации, когда все адаптивное разнообразие в популяции определяется одним аутосомным диаллельным локусом с аллеломорфами $A$ и $a$, что соответствует существованию в популяции трех генотипических групп $A A, A a$ и $a a$ с различающимися репродуктивными потенциалами. Будем предполагать, что фенотип особи жестко определяется ее генотипом; популяция панмиктична, в ней действуют менделевские правила наследования, а смежные поколения особей не перекрываются. Обозначим через $b_{A A}, b_{A a}$ и $b_{a a}$ репродуктивные потенциалы генотипов $A A, A a$ и $a а$ соответственно.

Уравнения динамики численности лимитированной популяции с неперекрывающимися поколениями и учетом генетического состава имеют вид:

$$
\begin{aligned}
& X_{n+1}=B_{n} Y_{n} \exp \left(-\alpha X_{n}-\beta Y_{n}\right), \\
& Y_{n+1}=s X_{n}, \\
& q_{n+1}=\frac{p_{n}\left(b_{A A} p_{n}+b_{A a}\left(1-p_{n}\right)\right)}{B_{n}}, \\
& p_{n+1}=q_{n} .
\end{aligned}
$$


Здесь $q_{n}$ - частота аллеля $A$ в младшем возрастном классе, $p_{n}$ - частота аллеля $A$ в старшем возрастном классе; $B_{n}=b_{A A} p_{n}{ }^{2}+2 b_{A a} p_{n}\left(1-p_{n}\right)+b_{a a}\left(1-p_{n}\right)^{2}-$ средний репродуктивный потенциал зрелого класса (средняя максимально возможная плодовитость особей с учетом выживаемости потомков) в году $n$. Все остальные обозначения переменных и параметров такие же, как и ранее.

Вывод уравнений (5) аналогичен выводу модели динамики структурированной популяции с учетом плотностно-зависимой регуляции роста и естественного отбора по величине репродуктивного потенциала в работе [Неверова и др., 2020]. Как видно, микроэволюционная модель (5) отличается от первоначальной модели (3) тем, что постоянный репродуктивный потенциал половозрелого класса $(B)$ заменен на перемененный средний репродуктивный потенциал $\left(B_{n}\right)$, для вычисления которого необходимо параллельно с моделью динамики численности рассматривать динамику частот аллелей, определяемую двумя дополнительными уравнениями.

При переходе к безразмерным переменным - относительным численностям $s \beta X \rightarrow x$ и $\beta Y \rightarrow y-$ модель (5) сводится к виду

$$
\left\{\begin{array}{c}
x_{n+1}=R_{n} y_{n} \exp \left(-\rho x_{n}-y_{n}\right), \\
y_{n+1}=x_{n}, \\
q_{n+1}=\frac{p_{n}\left(r_{A A} p_{n}+r_{A a}\left(1-p_{n}\right)\right)}{R_{n}} \\
p_{n+1}=q_{n},
\end{array}\right.
$$

где $\rho=\alpha /(s \beta), R_{n}=s b_{A A} p_{n}{ }^{2}+s 2 b_{A a} p_{n}\left(1-p_{n}\right)+s b_{a a}\left(1-p_{n}\right)^{2}=r_{A A} p_{n}{ }^{2}+2 r_{A a} p_{n}\left(1-p_{n}\right)+r_{a a}\left(1-p_{n}\right)^{2}$. Параметры $r_{A A}, r_{A a}$ и $r_{a a}$ в этом случае характеризуют репродуктивные потенциалы генотипов $A A$, $A a$ и $a a$ старшего возрастного класса с учетом выживаемости потомков до достижения зрелости, $R_{n}-$ средний репродуктивный потенциал в сезоне размножения $n$, параметр $\rho$ - относительный вклад младшей возрастной группы в лимитирование процесса воспроизводства.

\section{Стационарные точки модели (6) и их устойчивость}

Модель (6) имеет две мономорфные ненулевые по численности стационарные точки:

$$
\bar{x}=\frac{\ln r_{a a}}{1+\rho}, \bar{y}=\frac{\ln r_{a a}}{1+\rho}, \bar{q}=0, \bar{p}=0
$$

(имеет содержательный смысл при $r_{a a}>1$ ),

$$
\bar{x}=\frac{\ln r_{A A}}{1+\rho}, \quad \bar{y}=\frac{\ln r_{A A}}{1+\rho}, \quad \bar{q}=1, \quad \bar{p}=1
$$

(имеет содержательный смысл при $r_{A A}>1$ ), и одну полиморфную:

$$
\bar{x}=\bar{y}=\frac{1}{1+\rho} \ln \frac{r_{A a}^{2}-r_{A A} r_{a a}}{\left(2 r_{A a}-r_{A A}-r_{a a}\right)}, \quad \bar{q}=\frac{r_{A a}-r_{a a}}{2 r_{A a}-r_{A A}-r_{a a}}, \quad \bar{p}=\frac{r_{A a}-r_{a a}}{2 r_{A a}-r_{A A}-r_{a a}},
$$

которая имеет содержательный смысл, если выполняются следующие условия:

или

$$
r_{A a}>\max \left(r_{A A}, r_{a a}\right), \frac{r_{A a}{ }^{2}-r_{A A} r_{a a}}{2 r_{A a}-r_{A A}-r_{a a}}>1
$$

$$
r_{A a}<\min \left(r_{A A}, r_{a a}\right) \text { и } \frac{r_{A a}{ }^{2}-r_{A A} r_{a a}}{2 r_{A a}-r_{A A}-r_{a a}}>1 .
$$


Отметим, что уравнения, характеризующие изменение генетической структуры популяции, не зависят от ее численности, а результаты отбора по величине репродуктивного потенциала в экологически лимитированных популяциях не отличаются от результатов отбора по «фишеровским» приспособленностям в свободно размножающихся популяциях [Фрисман, Жданова, 2009; Неверова и др., 2020], в том смысле, что:

1) при $r_{i i}<1$ популяция вырождается;

2) промежуточные значения репродуктивного потенциала гетерозигот $-r_{A A}>r_{A a}>r_{a a}$ или $r_{a a}>r_{A a}>r_{A A}$ (движущий отбор) - приводят к установлению мономорфизма по аллелям, обеспечивающим больший репродуктивный потенциал;

3) повышенный репродуктивный потенциал гетерозигот $r_{A a}>\max \left(r_{a a}, r_{A A}\right)$ приводит к устойчивому полиморфизму;

4) пониженный репродуктивный потенциал гетерозигот $r_{A a}<\min \left(r_{a a}, r_{A A}\right)$ приводит к бистабильности мономорфных состояний.

Схематически результат отбора в зависимости от значений репродуктивных потенциалов различных генотипов можно представить так, как показано на рис. 2.

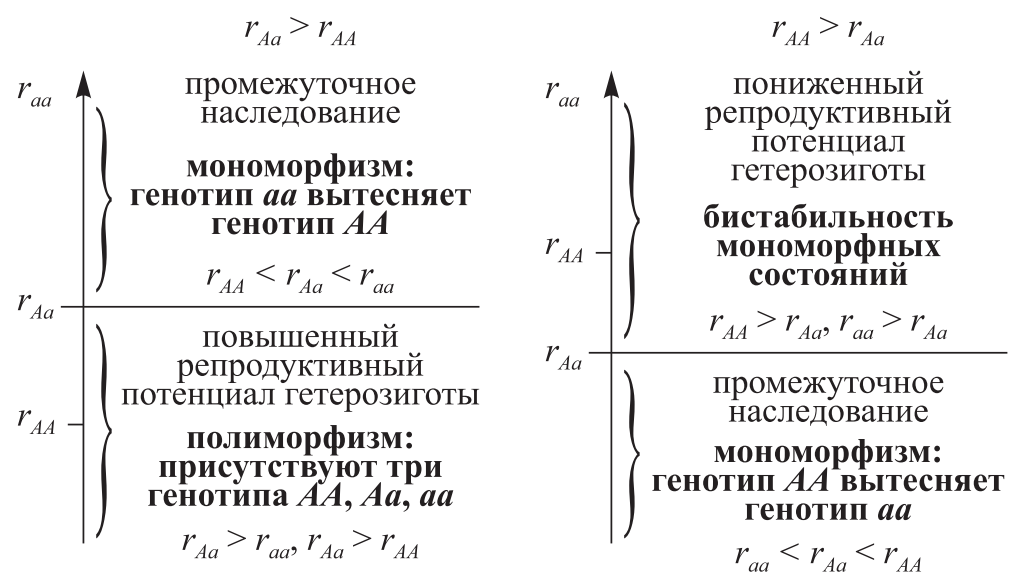

Рис. 2. Результат отбора в зависимости от соотношений репродуктивных потенциалов различных генотипов. По вертикальным осям откладываются значения репродуктивного потенциала гомозиготы $a a$ (т. е. $\left.r_{a a}\right)$, значения репродуктивного потенциала гомозиготы $A A\left(r_{A A}\right)$ и гетерозиготы $A a\left(r_{A a}\right)$ считаются фиксированными

Для того чтобы получить полное представление о влиянии значений популяционных параметров на устойчивость стационарных решений, а также о возникающих бифуркациях, происходящих в результате вариации значений коэффициентов, исследуем модель (6) на устойчивость, используя «треугольник» устойчивости, границы которого соответствуют линиям бифуркаций. Границы находятся на основе характеристического многочлена системы (6) $V=\lambda^{4}-S \lambda^{3}+H \lambda^{2}-L \lambda+J=0$ и задаются следующим образом [Кузнецов и др., 2012а].

Транскритическая бифуркация $(T), \lambda=1: H=S+L-J-1$.

Бифуркация удвоения периода $(P D), \lambda=-1: H=-S-L-J-1$.

Бифуркация Неймарка-Сакера $(N S), \lambda=e^{ \pm i \theta}:(S-L)(L-S J)=(1-J)^{2}(H-J-1)$. образом:

Для решения (7) коэффициенты характеристического многочлена задаются следующим

$$
S=\frac{\rho}{\rho+1} \ln \frac{1}{r_{a a}}, \quad H=-\frac{r_{a a}\left(1+\rho-\ln r_{a a}\right)}{r_{a a}(1+\rho)}+\frac{r_{A a}}{r_{a a}}, L=\frac{\rho \cdot r_{A a} \ln r_{a a}}{r_{a a}(1+\rho)}, \quad J=\frac{r_{A a}\left(1+\rho-\ln r_{a a}\right)}{r_{a a}(1+\rho)} .
$$

Коэффициенты характеристического многочлена для мономорфного решения (8) легко получить из коэффициентов полинома для решения (7), поскольку они симметричны относительно $r_{A A}$ и $r_{a a}$. 
Коэффициенты характеристического многочлена для полиморфного решения (9) имеют вид

$$
S=\frac{-\rho}{1+\rho} L n, \quad H=R+\frac{L n}{1+\rho}-1, L=\frac{-\rho}{1+\rho} R \cdot L n, \quad J=\frac{R(\operatorname{Ln}-1-\rho)}{1+\rho},
$$

где $L n=\ln \frac{r_{A a}^{2}-r_{A A} r_{a a}}{2 r_{A a}-r_{A A}-r_{a a}}, \quad R=\frac{2 r_{A A} r_{a a}-r_{A a}\left(r_{A A}+r_{a a}\right)}{r_{A a}^{2}-r_{A A} r_{a a}}$.

Примеры областей устойчивости мономорфных и полиморфного решений, формируемых бифуркационными линиями, представлены на рис. 3.

(a)

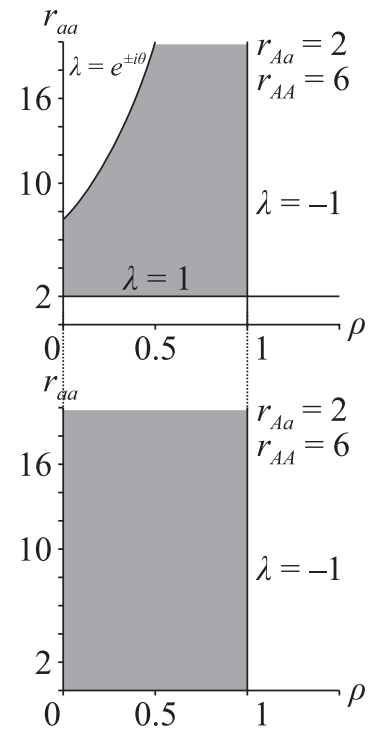

(в) 1.5

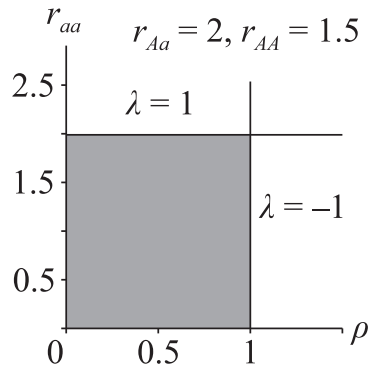

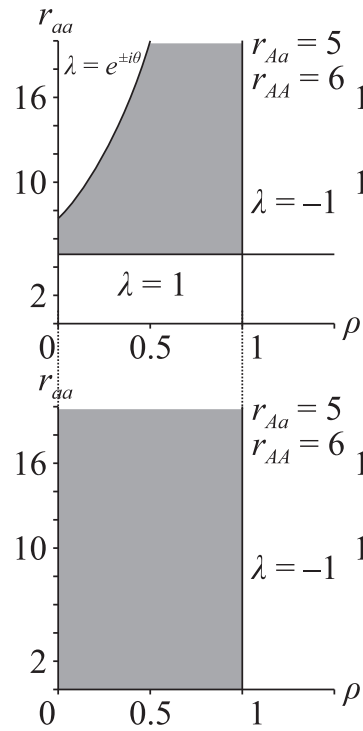
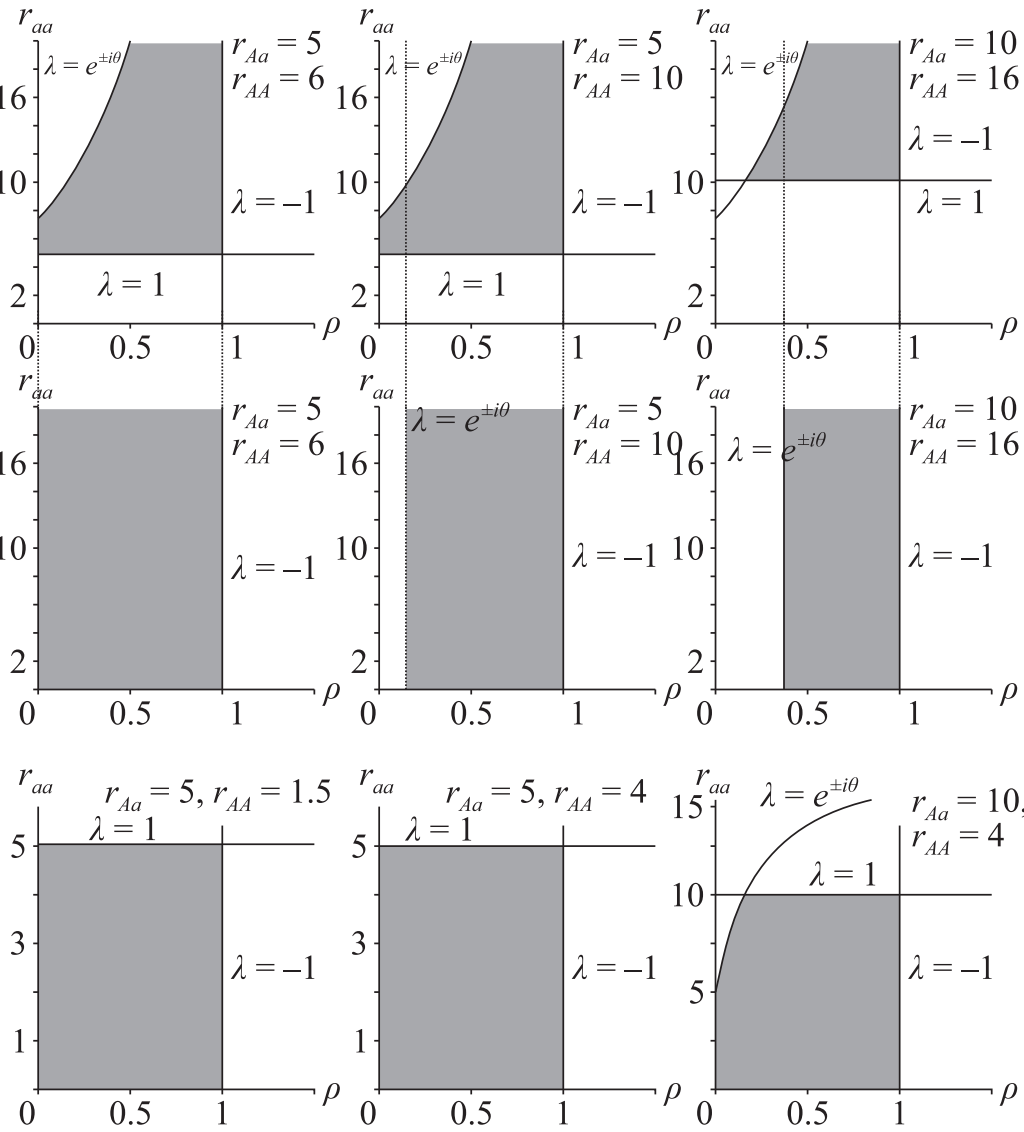

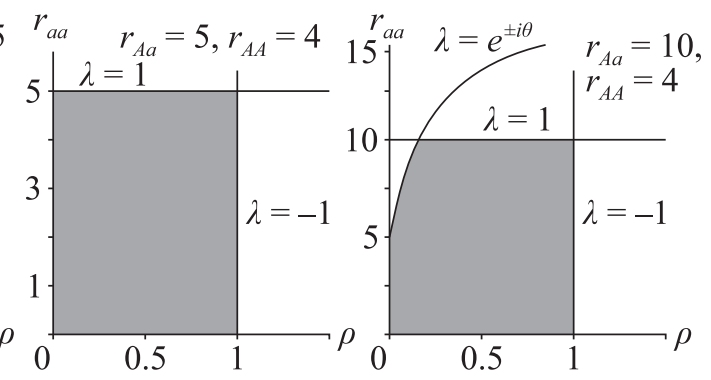

Рис. 3. Области устойчивости мономорфных решений (7) и (8) (ряды $a$ и 6 соответственно) и полиморфного решения (9) (ряд в) в плоскости параметров $\left(\rho, r_{a a}\right)$

Как видно на рис. 3, a, потеря устойчивости решения (7) реализуется как по сценарию Неймарка-Сакера, так и по сценарию Фейгенбаума. При этом способ потери устойчивости зависит от значений параметра $\rho$; так, если $\rho<1$, то потеря устойчивости сопровождается возникновением квазипериодических колебаний; если $\rho>1$, то наблюдается жесткий переход через мультипликатор -1 [Кузнецов и др., 2012а, 2012б]. Это совпадает с результатами исследования модели (4). Другими словами, если $r_{a a}>r_{A a}$ и $\rho<1$, то при переходе через границу $r_{a a}=e^{2(\rho+1)}$ потеря устойчивости происходит при комплексно-сопряженных корнях характеристического уравнения, причем генетический мономорфизм $a a$ сохраняется, но возникают колебания численности, сопровождающиеся появлением предельных инвариантных кривых, которые при дальнейшем удалении параметра $r_{a a}$ от границы устойчивости разрушаются с образованием весьма сложных предельных структур. Если $r_{a a}>r_{A a}$ и $\rho>1$, то потеря устойчивости происходит при переходе через мультипликатор - 1 . С ростом значений $r_{A a}$ область устойчивости мономорфного равновесия (7) сужается (рис. $3, a$ ) за счет смещения линии транскритиче- 
ской бифуркации. При этом границы, соответствующие субкритической бифуркации удвоения периода и бифуркации Неймарка-Сакера, остаются без изменений. Увеличение репродуктивного потенциала генотипа $A A$ также не изменяет параметрический портрет, соответствующий области устойчивости решения (7).

При $r_{A A} \leq e^{2}$ переход через границу $\rho=1$ ведет к возникновению субкритической бифуркации удвоения периода и, следовательно, потере устойчивости решения (8) с сохранением генетического мономорфизма $A A$. Отметим, что при $e^{2}<r_{A A}<e^{4}$ наблюдается возможность возникновения квазипериодических колебаний, поскольку потеря устойчивости решения (8) реализуется по сценарию Неймарка-Сакера (рис. 3 , б). В целом изменение значений репродуктивного потенциала гомозиготы $a a$ не влияет на динамические режимы мономорфного решения (8). В то время как рост репродуктивного потенциала гомозиготы $A A$ сужает область устойчивости неподвижной точки (8).

Полиморфное равновесие (9) также теряет устойчивость по двум сценариям (рис. 3, в). Отметим, что неподвижная точка (9) и режимы, возникшие в результате ее потери устойчивости, существуют при $0<r_{a a}<r_{A a}$. Причем чем больше значение $r_{A a}$, тем больше область устойчивости полиморфного равновесия. Анализ поведения границ области устойчивости полиморфной точки (9) в плоскости параметров $\left(\rho, r_{a a}\right)$ позволяет заключить, что если $r_{A a}>e^{2}$, то возникает возможность потери устойчивости по сценарию Неймарка-Сакера при $0<\rho<0.5 \ln r_{A a}-1$. Если $\rho>1$, то в полосе $0<r_{a a}<r_{A a}$ наблюдаются режимы динамики, возникшие в результате потери устойчивости полиморфного равновесия (9), вызванной переходом мультипликатора через -1 .

В зависимости от значений параметров $r_{A A}$ и $r_{A a}$ область устойчивости системы (6) представляет собой комбинацию областей устойчивости решений (7) и (8) при $r_{A A}>r_{A a}$ либо решений (7) и (9) при $r_{A A}<r_{A a}$.

При $r_{A A}<r_{A a}$ в полосе $r_{a a}<r_{A a}$ реализуется полиморфное равновесие, которое может терять устойчивость как через бифуркацию $\lambda=-1$, так и по сценарию Неймарка-Сакера (рис. 3,8 ). Следовательно, при $r_{A A}<r_{A a}$ с ростом значений параметра $r_{a a}$ сначала существует полиморфное равновесие (т. е. повышенный репродуктивный потенциал гетерозиготы, поскольку значения репродуктивных потенциалов обеих гомозигот меньше), которое затем сменяется мономорфным решением (7), поскольку генотип $a a$ с наибольшим репродуктивным потенциалом вытесняет остальные генотипы.

При $r_{A A}>r_{A a}$, как видно на рис. $3, a$, в области $r_{a a}>r_{A a}$ существует мономорфное решение $\bar{q}=0, \bar{p}=0$, которое может быть как устойчивым, так и неустойчивым. При $0<r_{a a}<r_{A a}$, будет реализовываться другое мономорфное решение $\bar{q}=1, \bar{p}=1$ (рис. 3, б). Более того, при $r_{a a}>r_{A a}$ можно говорить о бистабильности, поскольку область устойчивости решения (8), которой в плоскости параметров $\left(\rho, r_{a a}\right)$ соответствует полоса параллельная оси ординат (рис. $\left.3, \sigma\right)$, накладывается на область устойчивости решения (7).

В целом проведенное исследование демонстрирует, что итоговый генетический состав популяции (а именно, будет ли она устойчиво полиморфной) во многом определяется значениями репродуктивных потенциалов гетерозиготы и гомозигот. При этом появление (например, в результате мутаций) новых аллелей, обеспечивающих больший репродуктивный потенциал особей, приводит к естественному отбору этих аллелей и закономерному эволюционному росту среднего значения репродуктивного потенциала в популяции. Однако это увеличение среднего значения репродуктивного потенциала при плотностной регуляции рождаемости приводит к дестабилизации динамики численности возрастных групп.

\section{Мультистабильность динамики численности}

Для анализа возможных динамических режимов модели (6) были построены карты параметрических областей этих режимов. Карты получены следующим образом: в каждой точке (соответствующей одному пикселю) плоскости параметров выполнялось 5000 итераций ото- 
бражения (6), по результатам последних 500 шагов определялся период колебаний траектории, и эта точка окрашивалась в заданный цвет в соответствии с полученным периодом. Карты суммируют информацию не только о типе наблюдаемого динамического режима по численности (период наблюдаемых колебаний), но и генетическом составе, т. е. является ли популяция полиморфной или же мономорфной и относительно какого аллеля.

\section{Повышенный репродуктивный потенциал гетерозиготы и промежуточное доминирование при $\boldsymbol{r}_{A a}>r_{A A}$}

Полиморфизм в популяции возможен, только когда $r_{a a}<r_{A a}$ [Фрисман, Жданова, 2009; Неверова и др., 2020], поскольку в этом случае репродуктивный потенциал гетерозиготы оказывается выше репродуктивных потенциалов обеих гомозигот. В свою очередь, при $r_{a a}>r_{A a}$ реализуется промежуточное доминирование, в результате которого генотип $a a$ вытесняет все остальные генотипы, так как значение его репродуктивного потенциала наибольшее. Соответственно, при $r_{a a}>r_{A a}$ притягивающим оказывается решение $\bar{q}=0, \bar{p}=0$ или же режимы, возникшие в результате его потери устойчивости. Рис. 4 демонстрирует динамические режимы модели (6). Как видно, при $r_{a a}<r_{A a}$ существует полиморфное равновесие, причем чем больше значения $r_{A a}$, тем разнообразнее множество динамических режимов, возникающих в результате его потери устойчивости. При $r_{a a}>r_{A a}$ возникает мономорфное решение $\bar{q}=0, \bar{p}=0$, область устойчивости которого сужается с ростом значений $r_{A a}$. В целом видно, что потеря устойчивости неподвижных точек реализуется по двум сценариям. Примечательно, что увеличение репродуктивного потенциала гомозиготы $A A$ оказывает влияние преимущественно на режимы динамики полиморфной популяции: область устойчивости решения (9) сужается, и изменяется поведение бифуркационных линий (рис. 4, в). Также изменения касаются областей, где равновесия не устойчивы.

Отметим наличие областей мультистабильности, в которых реализация того или иного динамического режима зависит от значений начального условия. В частности, на картах рис. 4 можно наблюдать область 3-цикла, которая располагается поверх устойчивого равновесия и режимов, возникших в результате его потери устойчивости. Здесь вариация начального условия приведет к реализации либо 3-цикла, либо динамического режима «под ним». В наших предыдущих работах было показано, что в модели (4) без учета генетического разнообразия возникает мультистабильность, когда в зависимости от начальных условий возможна реализация как устойчивого равновесия, так и колебательных режимов динамики [Неверова, Фрисман, 2015; Neverova et al., 2018]. Как оказалось, в модели (6), учитывающей не только возрастную, но и генетическую структуры популяции, явление мультистабильности сохраняется.

$$
\begin{gathered}
r_{A a}=5, r_{A A}=4, \\
x_{0}=y_{0}=0.1,
\end{gathered}
$$

(a) $q_{0}=0.2, p_{0}=0.7$

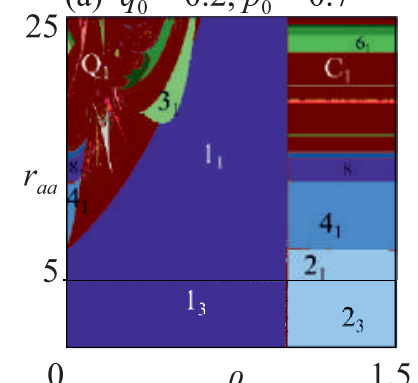

0

$$
\begin{gathered}
r_{A a}=15, r_{A A}=4, \\
x_{0}=y_{0}=0.1,
\end{gathered}
$$
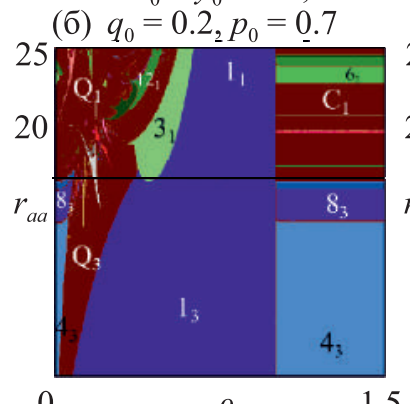

$\rho$

$$
\begin{gathered}
r_{A a}=20, r_{A A}=4, \\
x_{0}=y_{0}=0.1,
\end{gathered}
$$

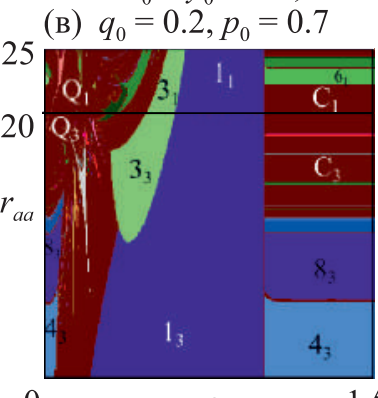

$\rho$

$$
\begin{gathered}
r_{A a}=20, r_{A A}=10, \\
x_{0}=y_{0}=0.1,
\end{gathered}
$$

(г) $q_{0}=0.2, p_{0}=0.7$

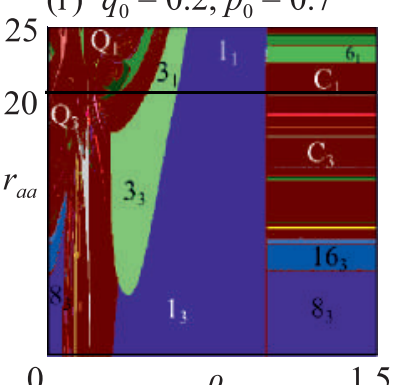

Рис. 4. Карты динамических режимов модели (6) в плоскости параметров $\left(\rho, r_{a a}\right)$. Числа соответствуют длине наблюдаемого цикла, индекс 1 отражает, что популяция мономорфна относительно аллеля $a$ $(\bar{q}=0, \bar{p}=0), 3$ - популяция полиморфна, $Q-$ квазипериодическая динамика, $C$ - хаотическая динамика 
Следует отметить, что сосуществующие устойчивые аттракторы могут весьма причудливо и сложно делить фазовое пространство модели между собой. Примеры структуры фазового пространства в областях мультистабильности для карты динамических режимов рис. 4, 8 представлены на рис. 5.
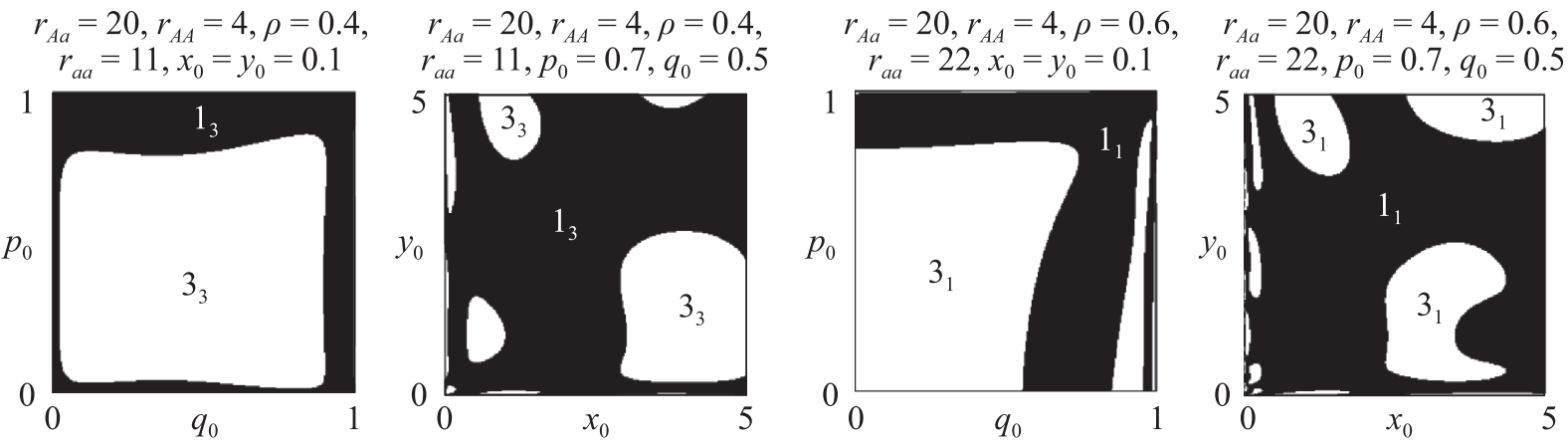

Рис. 5. Бассейны притяжения динамических режимов модели (6) по численности в области мультистабильности при повышенном репродуктивном потенциале гетерозиготы (режимы с индексом 3) и промежуточном доминировании (индекс 1). Числа соответствуют длине наблюдаемого цикла

На бассейнах притяжения в пространстве частот при полиморфизме видно, что при малой концентрации особей с новым генотипом вполне может произойти перескок в бассейн притяжения другого режима, в результате чего будет наблюдаться смена динамики численности. В целом анализ приведенных на рис. 5 бассейнов притяжения позволяет заключить, что смена динамического режима численности может произойти не только в случае вариации численностей возрастных групп в популяции, но и в результате изменения ее генетического состава. Отметим, что наличие мультистабильности (мультирежимности) принципиальным образом увеличивает количество и разнообразие возможных эволюционных сценариев и делает их зависимыми не только от параметров, но и от начальных условий, фаз циклов, при которых происходили соответствующие бифуркации, вызванные эволюционным ростом репродуктивного потенциала, и т. д.

\section{Неполное доминирование (промежуточное наследование) и пониженный репродуктивный потенциал гетерозиготы (ловушка бистабильности) при $r_{A a}<r_{A A}$}

Рассмотрим более подробно ситуацию, когда в модели (6) возникает бистабильность, т. е. одновременно существуют два устойчивых мономорфных равновесия. На рис. 6 представлены карты динамических режимов при $r_{A a}<r_{A A}$ в плоскости параметров $\left(\rho, r_{a a}\right)$, как видно, при $r_{a a}<r_{A a}$ наблюдается промежуточное доминирование, в результате которого побеждает генотип с наибольшим репродуктивным потенциалом $A A$ (рис. 6, a). Условие $r_{a a}>r_{A a}$ соответствует пониженному репродуктивному потенциалу гетерозиготы, при котором возникает бистабильность, и, следовательно, сосуществуют оба мономорфных равновесия. При этом какой из аттракторов является притягивающим в каждом конкретном случае определяется значениями начального условия. В частности, на картах видно, что выбор начального условия в области бистабильности определяет эволюционный путь, по которому будут развиваться популяция и, соответственно, асимптотический динамический режим.

Таким образом, если $r_{A A}>r_{A a}$, то при $r_{a a}<r_{A a}$ динамические режимы системы не зависят от начальных условий, здесь всегда развивается мономорфная популяция, особи которой имеют генотип $A A$. В области бистабильности при $r_{a a}>r_{A a}$ установившийся генетический состав зависит от начальных условий. Как и в предыдущем случае, здесь возникает мультирежимность: поверх области устойчивости неподвижной точки, располагается область 3-цикла, который рождается в результате касательной бифуркации [Фрисман и др., 2014; Neverova et al., 2018]. 

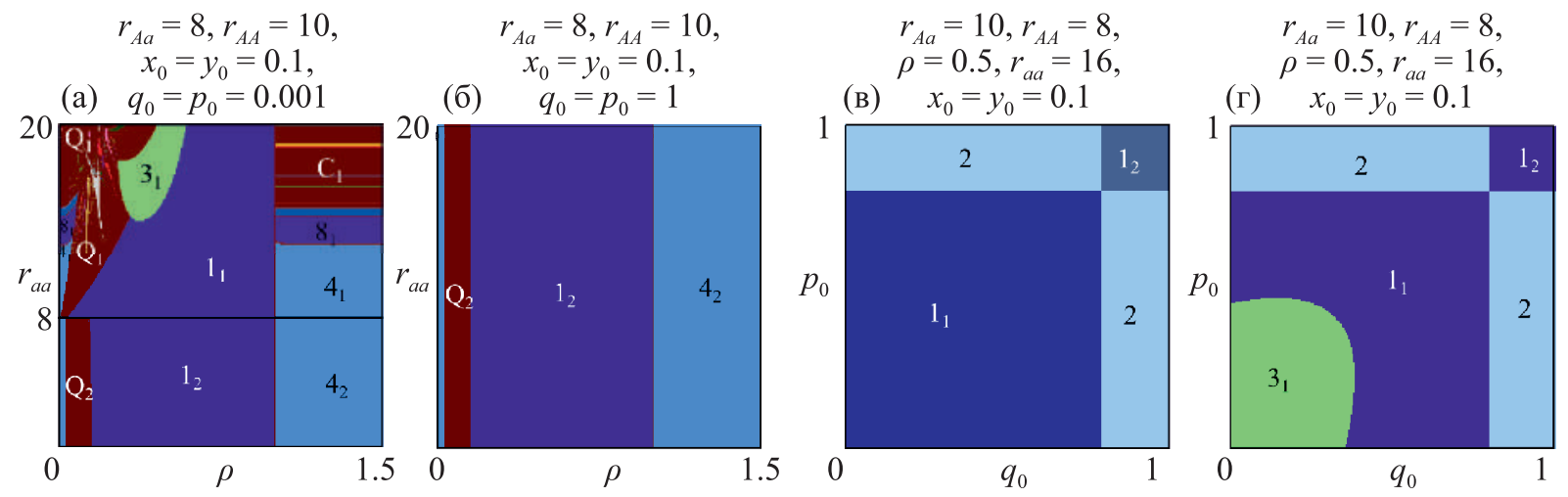

Рис. 6. а-б) Карты динамических режимов модели (6) в плоскости параметров $\left(\rho, r_{a a}\right)$ при вариации генетического состава популяции. Бассейны притяжения динамических режимов генетического состава (в) и численности (г). Числа соответствуют длине наблюдаемого цикла, индекс 1 отражает, что популяция мономорфна относительно аллеля $a(\bar{q}=0, \bar{p}=0), 2$ - популяция мономорфна относительно аллеля $A$ $(\bar{q}=1, \bar{p}=1), Q-$ квазипериодическая динамика

На рис. 6, в, 2 представлены бассейны притяжения динамических режимов частот генотипов и численностей для значений параметров из области мультистабильности. Как видно, устойчивое равновесие и 3-цикл делят между собой фазовое пространство, и, соответственно, изменения текущей численности популяции могут привести к изменениям режима динамики численности мономорфной популяции с генотипом $a a$. С другой стороны, случайные изменения генетического состава, вызванные вариацией текущей численности, могут привести к изменению направления эволюции, в результате чего генотип $A A$ вытеснит все остальные генотипы, и в перспективе будет развиваться мономорфная популяция с меньшим репродуктивным потенциалом.

Закономерным оказалось наличие областей притяжения 2-цикла, т. е. возможность третьего сценария развития популяции, а именно возникновение двухгодичных колебаний генетического состава, ведущего к колебаниям среднего значения репродуктивного потенциала и, следовательно, колебаниям численности популяции. Этот режим реализуется из сравнительно небольшой области начальных условий и заключается в циклических колебаниях генетической структуры, при которых в четные сезоны размножений в популяции преобладает один из аллелей, а в нечетные - другой.

\section{Колебания генетического состава: возникновение 2-цикла}

Для того чтобы изучить условия возникновения цикла длины 2, рассмотрим отображение, полученное после двукратного применения рекуррентных уравнений модели (6). Приходим к следующей системе уравнений:

$$
\left\{\begin{array}{c}
x_{n+2}=\left(r_{A A} q_{n}^{2}+2 r_{A a} q_{n}\left(1-q_{n}\right)+r_{a a}\left(1-q_{n}\right)^{2}\right) x_{n} \exp \left(-R_{n} y_{n} \rho \exp \left(-\rho x_{n}-y_{n}\right)-x_{n}\right), \\
y_{n+2}=R_{n} y_{n} \exp \left(-\rho x_{n}-y_{n}\right), \\
q_{n+2}=\frac{q_{n}\left(r_{A A} q_{n}+r_{A a}\left(1-q_{n}\right)\right)}{r_{A A} q_{n}^{2}+2 r_{A a} q_{n}\left(1-q_{n}\right)+r_{a a}\left(1-q_{n}\right)^{2}} \\
p_{n+2}=\frac{p_{n}\left(r_{A A} p_{n}+r_{A a}\left(1-p_{n}\right)\right)}{R_{n}} .
\end{array}\right.
$$

Поскольку два последних уравнения системы (11) не зависят от переменных $x$ и $y$, нетрудно получить следующие значения для частот генотипов в неподвижных точках этой 
системы:

$$
\begin{gathered}
\{\bar{q}=0, \bar{p}=0\}, \\
\{\bar{q}=1, \bar{p}=1\}, \\
\left\{\bar{q}=\frac{r_{A a}-r_{a a}}{2 r_{A a}-r_{A A}-r_{a a}}, \bar{p}=\frac{r_{A a}-r_{a a}}{2 r_{A a}-r_{A A}-r_{a a}}\right\}, \\
\{\bar{q}=1, \bar{p}=0\}, \quad\{\bar{q}=0, \bar{p}=1\}, \\
\left\{\bar{q}=\frac{r_{A a}-r_{a a}}{2 r_{A a}-r_{A A}-r_{a a}}, \bar{p}=0\right\},\left\{\bar{q}=0, \bar{p}=\frac{r_{A a}-r_{a a}}{2 r_{A a}-r_{A A}-r_{a a}}\right\}, \\
\left\{\bar{q}=\frac{r_{A a}-r_{a a}}{2 r_{A a}-r_{A A}-r_{a a}}, \bar{p}=1\right\},\left\{\bar{q}=1, \bar{p}=\frac{r_{A a}-r_{a a}}{2 r_{A a}-r_{A A}-r_{a a}}\right\} .
\end{gathered}
$$

Значения частот (12), (13) и (14) соответствуют мономорфным и полиморфной стационарным точкам (7), (8) и (9) системы (6) и 2-циклам этой системы, в которых генетический состав постоянный, а численность колеблется с периодом 2. Такие циклы возникают, когда соответствующие неподвижные точки (7), (8) или (9) теряют устойчивость через бифуркацию удвоения периода: популяция остается постоянна по генетического составу, но ее численность совершает двухгодичные колебания.

Значения (15), (16) и (17) соответствуют значениям частот аллелей в 3 разных парах 2-циклов системы (6). Циклы каждой пары имеют одинаковые значения переменных, но разные значения фаз. Отметим, что эти противофазные циклы существует одновременно и их области устойчивости совпадают. Однако какой из них окажется притягивающим, определяется начальными условиями. Следует подчеркнуть, что циклы (15) возникают при пониженном репродуктивном потенциале гетерозиготы, в то время как циклы (16) и (17) могут реализоваться только при полиморфизме.

Характеристический многочлен системы (11) имеет вид

$$
\left(X_{x}^{\prime} Y_{y}^{\prime}-X_{y}^{\prime} Y_{x}^{\prime}-\lambda\left(X_{x}^{\prime}+Y_{y}^{\prime}\right)+\lambda^{2}\right)\left(Q_{q}^{\prime}-\lambda\right)\left(P_{p}^{\prime}-\lambda\right)=0,
$$

где

$$
\begin{gathered}
X=R(q) x \exp (-R(p) y \rho \exp (-\rho x-y)-x), \quad Y=R(p) y \exp (-\rho x-y), \\
Q=q\left(r_{A A} q+r_{A a}(1-q)\right) / R(q), \quad P=p\left(r_{A A} p+r_{A a}(1-p)\right) / R(p), \\
R(p)=r_{A A} p^{2}+2 r_{A a} p(1-p)+r_{a a}(1-p)^{2}, \quad R(q)=r_{A A} q^{2}+2 r_{A a} q(1-q)+r_{a a}(1-q)^{2} .
\end{gathered}
$$

Нетрудно показать, что для решений (15) справедливо $Q_{q}^{\prime}(\bar{q}=1, \bar{p}=0)=r_{A a} / r_{a a}$, $P_{p}^{\prime}(\bar{q}=1, \bar{p}=0)=r_{A a} / r_{A A}$ и $Q_{q}^{\prime}(\bar{q}=0, \bar{p}=1)=r_{A a} / r_{A A}, P_{p}^{\prime}(\bar{q}=0, \bar{p}=1)=r_{A a} / r_{a a}$. Соответственно, при полиморфизме $r_{A a}>\max \left\{r_{A A}, r_{a a}\right\}$ решения (15) неустойчивы. При промежуточном доминировании, когда $r_{a a}<r_{A a}<r_{A A}$ или $r_{A A}<r_{A a}<r_{a a}$, решения (15) также неустойчивы, однако соответствующие 2-циклы системы (6) вполне могут существовать, поскольку происходящие в субпопуляциях четных и нечетных лет генетические мутации оказываются независимыми и не переходят из одной субпопуляции в другую. В силу этого, если в какой-то момент $(n=0)$ в мономорфной по одной из гомозигот, например $a a$, общей (состоящей из двух субпопуляций) популяции произойдет положительная мутация $A$, такая, что $r_{A A}<r_{A a}<r_{a a}$, то эта мутация затронет только одну из субпопуляций: $q_{0}>0$ при $p_{0}=0$. Поскольку согласно (11) вторые итерации переменных $p$ и $q$ происходят независимо, то $q$ четных лет будет расти и затем стремиться к 1 , а $p$ останется равным 0 , и в результате мы будем наблюдать 2 -цикл с $\{\bar{q}=1, \bar{p}=0\}$. Этот цикл достигается только при $p_{0}=0$, и поэтому он неустойчив: попадание любого количества аллеля $A$ в субпопуляцию приведет к его закреплению. Однако реальный механизм такого 
«попадания» - это мутация, аналогичная произошедшей в смежной субпопуляции, что крайне маловероятно. По-видимому, именно независимость генетической эволюции субпопуляций четных и нечетных лет является основным источником различия в генетическом составе и численности этих субпопуляций.

В случае пониженного репродуктивного потенциала гетерозиготы $r_{A a}<\min \left\{r_{A A}, r_{a a}\right\}$ peшения (15) устойчивы.

Рассмотрим теперь неподвижные точки (16). Если $r_{A a}>r_{a a}$, то эти решения неустойчивы, поскольку $\quad Q_{q}^{\prime}\left(\bar{q}=0, \bar{p}=\frac{r_{A a}-r_{a a}}{2 r_{A a}-r_{A A}-r_{a a}}\right)=\frac{r_{A a}}{r_{a a}}>1 \quad$ и $\quad P_{p}^{\prime}\left(\bar{q}=\frac{r_{A a}-r_{a a}}{2 r_{A a}-r_{A A}-r_{a a}}, \bar{p}=0\right)=\frac{r_{A a}}{r_{a a}}>1$.

Отметим, что при $r_{A a}<r_{A A}$ решения (16) теряют физический смысл, поскольку репродуктивный потенциал гетерозиготы пониженный. Следовательно, при $r_{A a}>\max \left\{r_{A A}, r_{a a}\right\}$ реализация данного решения возможна, только если одна из частот будет равна нулю. Отметим, что попадание в данное неустойчивое состояние может оказаться вполне осуществимым, если в одном из поколений общей мономорфной по $a a$ популяции произойдет мутация $A$ такая, что $r_{A a}>\max \left\{r_{A A}, r_{a a}\right\}$.

В свою очередь, если $r_{A a}>\max \left\{r_{A A}, r_{a a}\right\}$, что обеспечивает существование полиморфизма, то при $r_{A a}>r_{a a}$ решения (17) также неустойчивы, поскольку $Q_{q}^{\prime}\left(\bar{q}=1, \bar{p}=\frac{r_{A a}-r_{a a}}{2 r_{A a}-r_{A A}-r_{a a}}\right)=\frac{r_{A a}}{r_{A A}}>1$, $P_{p}^{\prime}\left(\bar{q}=\frac{r_{A a}-r_{a a}}{2 r_{A a}-r_{A A}-r_{a a}}, \bar{p}=1\right)=\frac{r_{A a}}{r_{A A}}>1$, и, следовательно, как и в предыдущем случае, реализация этого решения возможна, только если одна из частот будет равна не изменяющейся координате неподвижной точки, т. е. единице (другими словами, в одном из поколений общей мономорфной по $A A$ популяции произойдет мутация $a$ такая, что $\left.r_{A a}<\min \left\{r_{A A}, r_{a a}\right\}\right)$.

Численности разных возрастных групп в неподвижных точках для соответствующих $\bar{q}$ и $\bar{p}$ находятся на основе двух первых уравнений системы (11). Как оказалось, каждому стационарному генетическому составу в дополнение может быть поставлен один из трех наборов соответствующих стационарных значений численности:

$$
\begin{gathered}
\left\{\bar{x}=\frac{\ln R(\bar{q})-\rho \ln R(\bar{p})}{1-\rho^{2}}, \bar{y}=\frac{\ln R(\bar{p})-\rho \ln R(\bar{q})}{1-\rho^{2}}\right\}, \\
\{\bar{x}=\ln R(\bar{q}), \bar{y}=0\}, \\
\{\bar{x}=0, \bar{y}=\ln R(\bar{p})\},
\end{gathered}
$$

где $R(\bar{q})=r_{A A} \bar{q}^{2}+2 r_{A a} \bar{q}(1-\bar{q})+r_{a a}(1-\bar{q})^{2}, R(\bar{p})=r_{A A} \bar{p}^{2}+2 r_{A a} \bar{p}(1-\bar{p})+r_{a a}(1-\bar{p})^{2}$.

Отметим, что в случае равновесного генетического мономорфизма по гомозиготе $a a$, определяемого (12), нетривиальное по численности решение совпадает с неподвижной точкой (7), т. е. это стационарное состояние. Полутривиальные по численности решения соответствуют противофазным 2-циклам, возникшим в результате потери устойчивости равновесия (7) при переходе собственного числа через -1 , т. е. через прямую $\rho=1$ (когда $r_{a a}<e^{2}$ ). При этом области устойчивости двух противофазных 2-циклов совпадают, и какой из них окажется притягивающим, определяется начальными условиями, а неустойчивая точка (7) лежит на границе бассейнов притяжения. Для равновесного мономорфизма по гомозиготе $a a$, определяемого (13), все аналогично. Области устойчивости решений (12) и (13) представлены на рис. 7, $a, \sigma$. Границы области устойчивости найдены на основе «треугольника устойчивости» [Кузнецов и др., $2012 \mathrm{a}, 20126]$. Устойчивый 2-цикл (12) реализуется в области $\rho>1$ и $r_{A a}<r_{a a}<e^{2}$ независимо от значений репродуктивного потенциала гомозиготы $A A$ (рис. 7, a). 2-цикл (13) в плоскости параметров $\left(r_{a a}, \rho\right)$ устойчив в полуплоскости $\rho>1$, если $r_{A A}<e^{2}$ (рис. 7, б). «Генетическое разнообразие» (14) устойчиво в полосе $0<r_{a a}<r_{A a}$ при $\rho>1$, если $r_{A A}<r_{A a}$ и $r_{A a}<e^{2}$, что хорошо вид- 
но на рис. $4, a$. При равновесных значениях частот аллелей, задаваемых равенствами (15), система (11) имеет три разные пары стационарных решений:

$$
\begin{gathered}
\left\{\bar{x}=\frac{\ln r_{A A}-\rho \ln r_{a a}}{1-\rho^{2}}, \bar{y}=\frac{\ln r_{a a}-\rho \ln r_{A A}}{1-\rho^{2}}, \bar{q}=1, \bar{p}=0\right\} \text { и } \\
\left\{\bar{x}=\frac{\ln r_{a a}-\rho \ln r_{A A}}{1-\rho^{2}}, \bar{y}=\frac{\ln r_{A A}-\rho \ln r_{a a}}{1-\rho^{2}}, \bar{q}=0, \bar{p}=1\right\}, \\
\left\{\bar{x}=0, \bar{y}=\ln r_{a a}, \bar{q}=1, \bar{p}=0\right\} \text { и }\left\{\bar{x}=\ln r_{a a}, \bar{y}=0, \bar{q}=0, \bar{p}=1\right\}, \\
\left\{\bar{x}=\ln r_{A A}, \bar{y}=0, \bar{q}=1, \bar{p}=0\right\} \text { и }\left\{\bar{x}=0, \bar{y}=\ln r_{A A}, \bar{q}=0, \bar{p}=1\right\},
\end{gathered}
$$

которые определяют значения переменных в противофазных 2-циклах модели (6). Области устойчивости решений (12), (13) и (18)-(20) представлены на рис. 7.

(a)
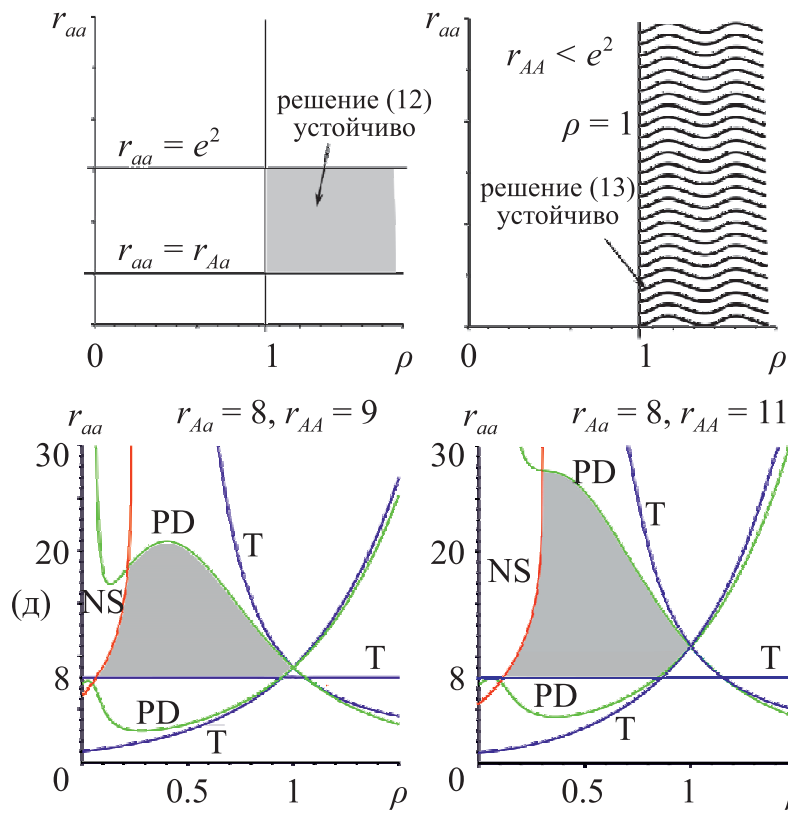

(б)

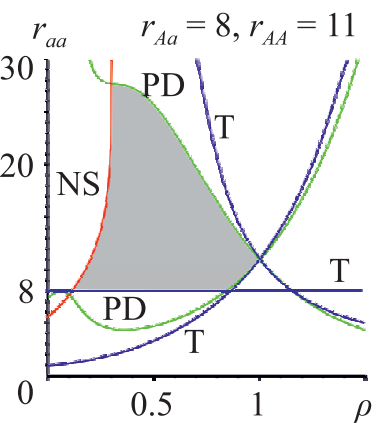

(в)

(г)

Рис. 7. Область устойчивости двухгодичных колебаний мономорфной популяции с генотипом $a a$ (a) и $A A$ (б). в) Области устойчивости решений (19) и (20). г) Деление параметрического пространства решениями (18)-(20): в области I реализуются решение (18) и режимы, возникшие в результате его потери устойчивости. В областях II и III реализуются решения (19) и (20) и/или режимы, возникшие в результате их потери устойчивости, соответственно. д) Область устойчивости 2-цикла (18) при вариации значений параметров

Линии транскритической бифуркации и удвоения периода для решения (19) имеют вид $r_{a a}=r_{A A}{ }^{\rho}, r_{A A}=e^{2}$ соответственно. Для решения (20) условия симметричны относительно $r_{a a}$ и $r_{A A}$, т. е. $r_{A A}=r_{a a}{ }^{\rho}, r_{a a}=e^{2}$. 2-циклы (19) и (20) устойчивы, если $r_{a a}<e^{2}$ и $r_{A A}<e^{2}$ соответственно (рис. 7, 6). Область устойчивости решения (18) представлена на рис. 7,. . Как видно, при $r_{A A}=r_{a a}$ линии касательной бифуркации и удвоения периода пересекаются в одной точке, то есть возникает fold-flip-бифуркация, в результате которой рождается 2-цикл. Данный 2-цикл бифурцирует как по сценарию Фейгенбаума (переход через линию $P D$ ), так и по сценарию Неймарка-Сакера (линия $N S$ ). При этом видно, что рост значений репродуктивного потенциала гомозиготы $A A$ расширяет область устойчивости 2-цикла. В то время как увеличение репродуктивного потенциала гетерозиготы уменьшает область устойчивости 2-цикла за счет смещения линии касательной бифуркации (T). 
В целом же параметрическое пространство в области значений параметров $r_{a a}>r_{A a}$ относительно решений (18)-(20) делится на три части, в каждой из которых частоты аллелей меняются одинаково в соответствии с (15), но разные сценарии развития популяции, возникшие в результате потери устойчивости разных неподвижных точек (рис. 7, 2). На основе какой неподвижной точки будет эволюционировать динамический режим, определяется значениями популяционных параметров. Так, в области I реализуются решение (18) и режимы, возникшие в результате его потери устойчивости (рис. 7, 2). В областях II и III реализуются решения (19) и (20) и/или режимы, возникшие в результате их потери устойчивости, соответственно.

На рис. 8 представлены карты динамических режимов модели (6), демонстрирующие деление параметрического пространства разными предельными решениями. На этих параметрических портретах выделяются (светло-голубые) области устойчивости разных 2-циклов. Далее на рисунке приведены траектории, демонстрирующие динамику численностей возрастных классов и частот аллелей при значениях параметров из областей устойчивости различных 2-циклов. Как видно, при выбранном начальном условии на рис. $8, a$ выделяются 4 области с различающейся динамикой двухгодичных колебаний (рис.8, в).

(a) $r_{A a}=4, r_{A A}=7$
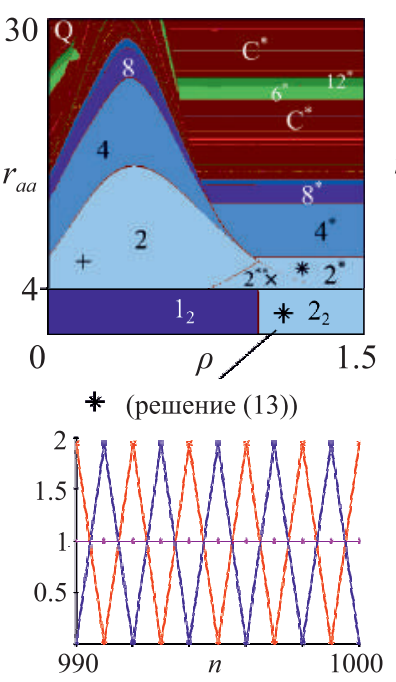

область устойчивости 2-цикла
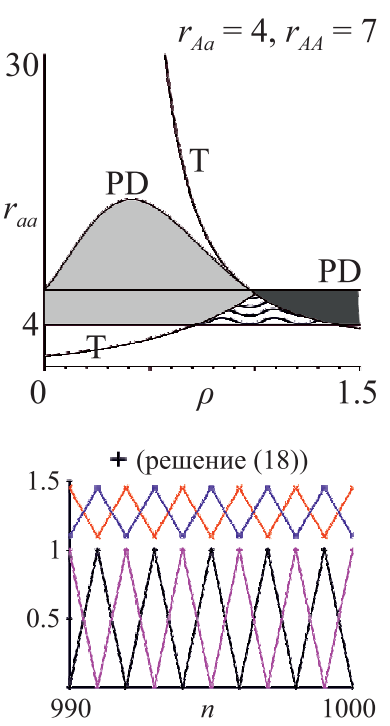

(18) (б) $r_{A a}=4, r_{A 4}=7$
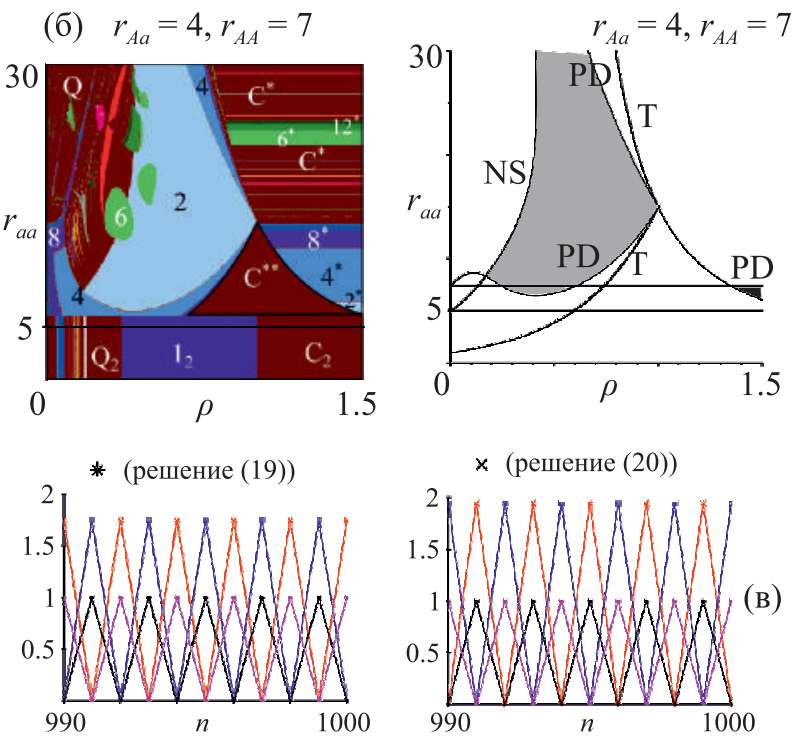

$\times$ (решение (20))

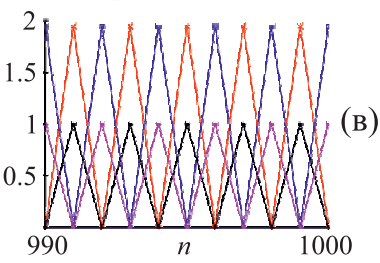

(B)

Рис. 8. а, б) Карты динамических режимов системы (6) при начальном условии $x_{0}=0.01, y_{0}=1, q_{0}=0.9$, $p_{0}=0.1$, дополненные параметрическими портретами. Числа соответствуют длине наблюдаемого цикла, $Q$ - квазипериодическая динамика, $C$ - хаотическая динамика. Нижний индекс 2 отражает, что популяция мономорфна по аллелю $A$, в областях без нижнего индекса частоты аллелей совершают двухгодичные колебания. Режимы с верхними индексами * и ** соответствуют решениям (19) и (20) и режимам, возникшим в результате их потери устойчивости, соответственно. Отсутствие верхнего индекса соответствует решению (18). (в) Траектории модели (6) при значениях параметров из областей устойчивости 2-циклов карты (а)

Согласно рис. 7 и 8 2-циклы (18)-(20) могут существовать при значениях параметра $\rho$ как больше, так и меньше единицы. При $\rho>1$ реализуются решения (19) и (20), которые могут быть как устойчивыми, так и нет (рис. $8, a, \sigma)$. С другой стороны, при $\rho>1$ могут существовать устойчивые двухгодичные колебания численности мономорфной популяции. Так, на рис. $8, a$ реализуется устойчивое решение (13). При этом в силу выбранного начального условия мы не наблюдаем цикл (12), однако он существует и устойчив, поскольку $r_{A a}=4<e^{2}$ (рис. 7, $a$ ). Следовательно, в случае пониженного репродуктивного потенциала гетерозиготы возможны ситуации, когда сосуществуют несколько устойчивых 2-циклов. В частности, при $\rho>1, r_{a a}<e^{2}$ 
и $r_{A A}<e^{2}$ в полосе $r_{A a}<r_{a a}<e^{2}$ сосуществуют три разных устойчивых 2-цикла, каждый из которых характеризуется своей структурой колебаний частот аллелей (рис. 7, $a-8$ ). Если $\rho>1$, $r_{a a}<e^{2}$ и $r_{A A}>e^{2}$, тогда в полосе $r_{A a}<r_{a a}<e^{2}$ только при $r_{a a}>r_{A A}{ }^{\rho}$ сосуществуют 2-циклы (12), (13) и (18), а при $r_{A a}>e^{2}$ устойчивых 2-циклов нет.

Однако, даже если $r_{A a}>e^{2}$, существует область параметрического пространства, где 2 -цикл (18) устойчив (рис. 7,8 ). Отметим, что этот 2 -цикл реализуется при $\rho<1$, и, следовательно, в этой области нет других устойчивых 2-циклов, поскольку $r_{A a}>e^{2}$.

\section{Сценарии развития при пониженном репродуктивном потенциале гетерозиготы: бистабильность и мультистабильность}

Проведенное исследование позволяет заключить, что при пониженном репродуктивном потенциале гетерозиготы при одних и тех значениях демографических параметров возможны 3 пути развития популяции. Это связано с тем, что при $r_{A a}<\min \left\{r_{A A}, r_{a a}\right\}$ друг на друга накладываются области устойчивости следующих решений: двух мономорфных (7), (8) и циклического (15). Какое именно из этих решений является притягивающим, определяется начальными условиями, а для реализуемой траектории - текущим состоянием популяции. Для того чтобы продемонстрировать данную мультистабильность, на рис. 9 представлены карты динамических режимов при различных начальных условиях.

Отметим, что в области бистабильности мономорфных решений отображение (11) по переменным $p$ и $q$ для решения (15) всегда оказывается сжимающим, и, следовательно, колебания генетического состава, задающие 2-цикл, существуют и устойчивы при любых значениях параметров в области пониженного репродуктивного потенциала гетерозиготы $r_{A a}<\min \left\{r_{A A}, r_{a a}\right\}$. Это легко доказать, исследовав на устойчивость подсистему, описывающую динамику генетического состава, модели (11). В целом же параметрический портрет, отражающий динамические режимы генетического состава при различных значениях репродуктивных потенциалов гомозигот, можно представить в виде следующей схемы (рис. 10, a). На рис. 10, a явно выделяется область, в которой устойчивы три варианта генетического разнообразия популяции. Соответственно, в случае пониженного репродуктивного потенциала гетерозиготы возникающий двухгодичный цикл по переменным $p$ и $q$ устойчив всегда, при этом двухгодичные колебания численности популяции могут бифурцировать как по сценарию Фейгенбаума, так и по сценарию Неймарка-Сакера (рис. 10, б). Отметим наличие областей мультистабильности, в которых перекрываются области циклов с разными периодами, в частности, этим объясняется нарушение симметрии на карте $10, \sigma$.

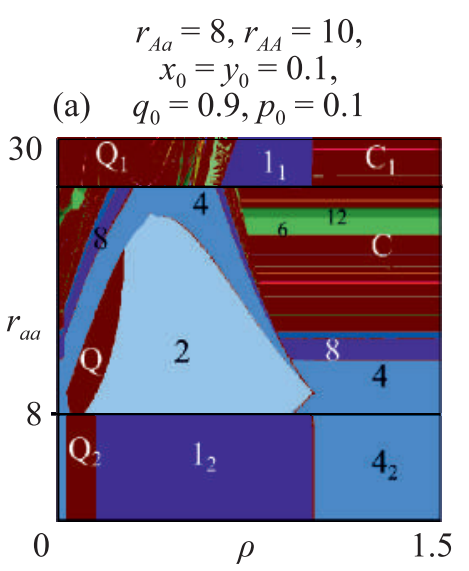

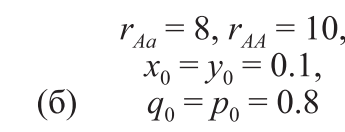

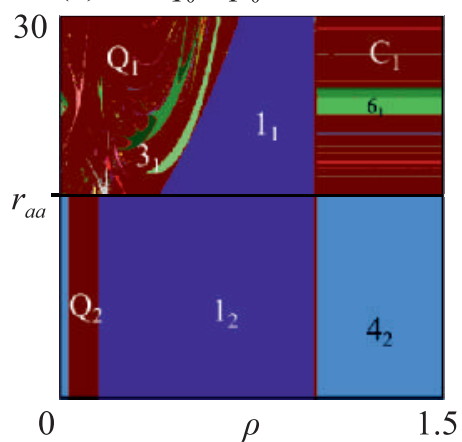

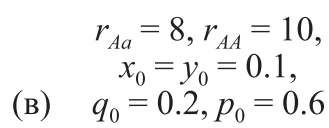

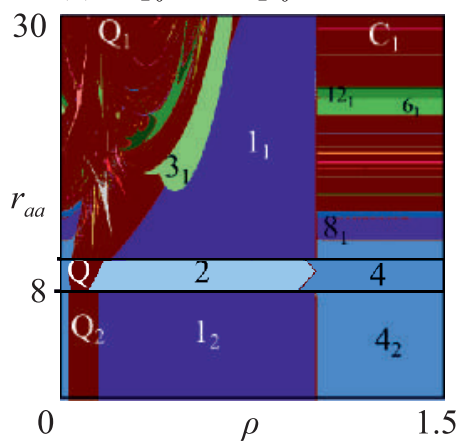

Рис. 9. Карты динамических режимов системы (6) при различных начальных условиях. Числа соответствуют длине наблюдаемого цикла, $Q$ - квазипериодическая динамика, $C$ - хаотическая динамика. Индекс 1 отражает, что популяция мономорфна по аллелю $a, 2$ - популяция мономорфна по аллелю $A$, в областях без индекса генетический состав совершает двухгодичные колебания 
(a)

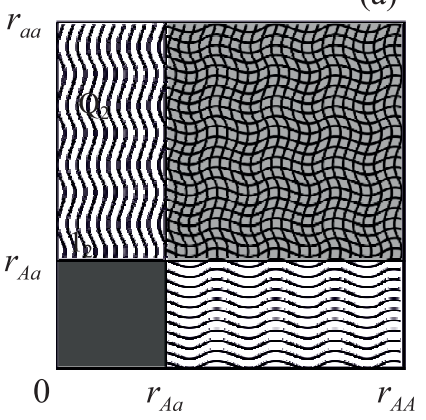

(г) $r_{A a}=5, \rho=0.3$

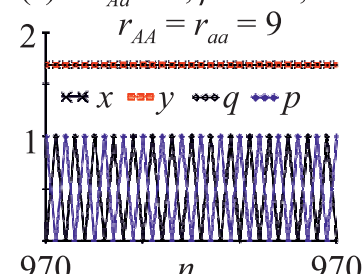

(б) $r_{A a}=5, \rho=0.3$

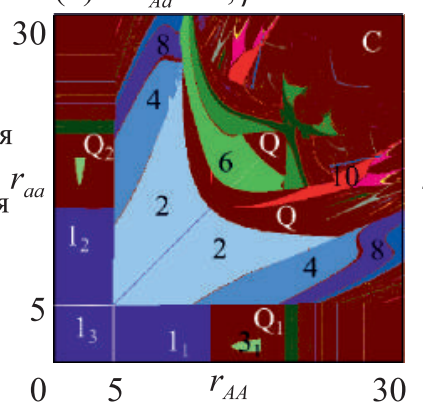

(e) $r_{A a}=5, \rho=0.3$

(д) $r_{A a}=5, \rho=0.3$,

2 $r_{A A}=9.5, r_{a a}=9$

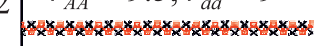

$* * x=0=y \quad \cos q \cdots p$
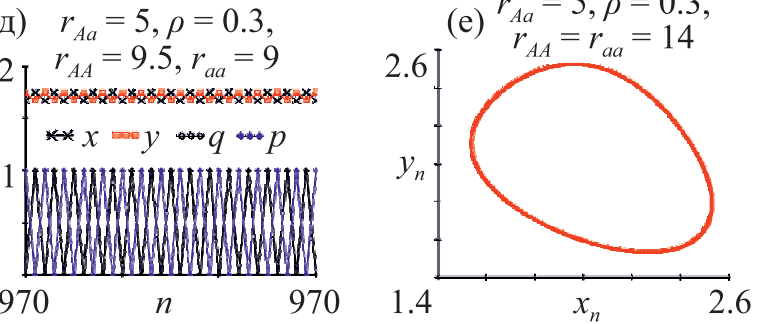

(в) $r_{A a}=5, \rho=0.5$

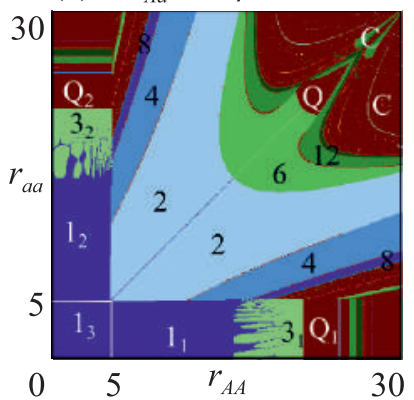

$r=5, \rho=0.3$

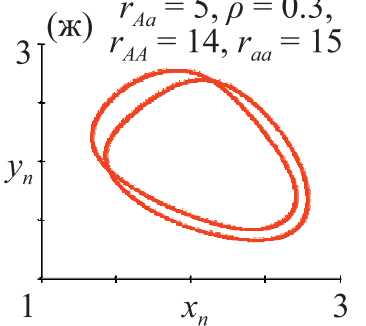

Рис. 10. Режимы динамики генетического (а) состава и численности популяции (б-в), дополненные траекториями (г-д) и фазовыми портретами (е-ж) модели (6) для начального условия $x_{0}=y_{0}=1, q_{0}=0.99$, $p_{0}=0.01$. Числа соответствуют длине наблюдаемого цикла. Индекс 1 отражает, что популяция мономорфна по аллелю $a, 2$ - популяция мономорфна по аллелю $A$, в областях без индекса генетический состав совершает двухгодичные колебания

В случае возникновения двухгодичных колебаний генетического состава режим динамики численности представляет собой наложение двух аттракторов, характерных для ведущих генотипов, каждой из субпопуляций смежных поколений. Однако численность популяции может стабилизироваться, если значения репродуктивных потенциалов гомозигот равны (рис. 10, г). В частности, на картах рис. 10 видно, что на диагонали, соответствующей случаю равенства репродуктивных потенциалов гомозигот, два аттрактора - элементы 2-цикла - совпадают, и, как результат, популяция демонстрирует стабильную динамику. Если же репродуктивные потенциалы гомозигот отличаются, даже не значительно, колебания генетического состава сопровождаются колебаниями численностей возрастных классов (рис. 10, $\partial$ ). То же самое наблюдается в области цикла с периодом 6 , при равенстве потенциалов гомозигот реализуется 3-цикл. В свою очередь, в области квазипериодической динамики при $r_{a a}=r_{A A}$ два аттрактора совпадают, и наблюдается одна инвариантная кривая (рис. 10, e, ж).

Деление фазового пространства модели сосуществующими динамическими режимами относительно генетического состава популяции представлено на рис. 11. Как видно, в пространстве частот при $r_{A A}=r_{a a}$ бассейны притяжения мономорфных равновесий равны и симметрично расположены, и их между собой разделяет область, соответствующая колебаниям генетического состава с периодом 2 (рис. $11, a$ ). При разных репродуктивных потенциалах гомозигот область притяжения мономорфного равновесия для гомозиготы с большим репродуктивным потенциалом больше, чем для гомозиготы с меньшим репродуктивным потенциалом (рис $11,6,6)$. Рост репродуктивного потенциала гетерозиготы приводит к увеличению бассейна притяжения мономорфного решения с большим репродуктивным потенциалом и сужению областей притяжения второго мономорфного состояния и двухгодичных колебаний генетического состава, вплоть до их полного исчезновения при $r_{A a}=\min \left\{r_{a a}, r_{A A}\right\}$ (рис. 11, г).

Нетрудно показать, что множество начальных условий, соответствующих частотам генетического состава популяции, в зависимости от асимптотического генетического распределения может быть поделено следующим образом:

1) мономорфизм относительно аллеля $a(\bar{q}=0, \bar{p}=0)$ при $q_{0}, p_{0}<p^{*}$;

2) мономорфизм относительно аллеля $A(\bar{q}=1, \bar{p}=1)$ при $q_{0}, p_{0}>p^{*}$; 
(a) $r_{A a}=5, r_{A A}=9, r_{a a}=9$, $\rho=0.3, x_{0}=y_{0}=1$

(б) $r_{A a}=5, r_{A A}=9, r_{a a}=14$

(в) $r_{A a}=5, r_{A 4}=14, r_{a a}=9$,

(г) $r_{A a}=7, r_{A 4}=14, r_{a a}=9$,

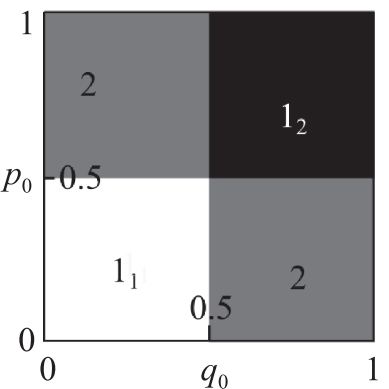
$\rho=0.3, x_{0}=y_{0}=1$
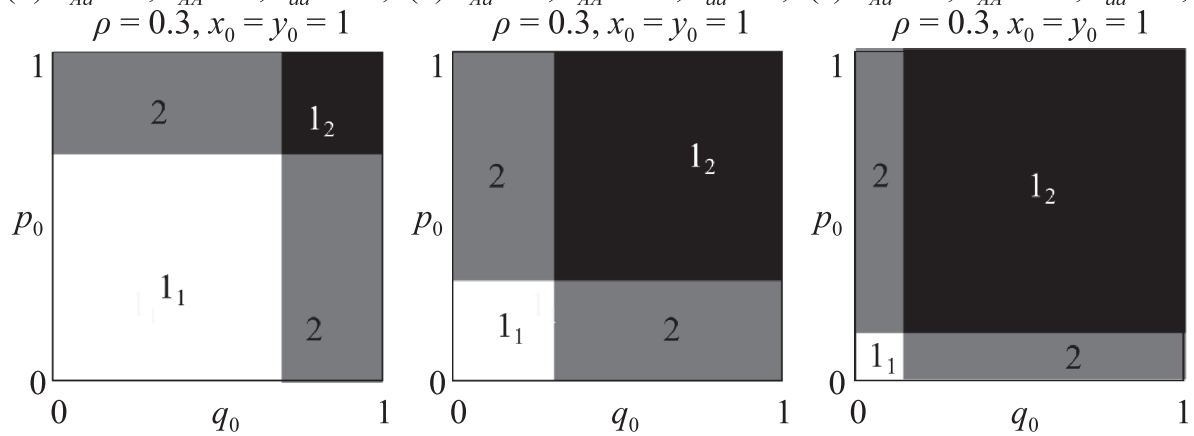

Рис. 11. Бассейны притяжения динамических режимов модели (6), соответствующие генетическому составу популяции, в области бистабильности. Числа соответствуют длине наблюдаемого цикла. Индекс 1 отражает, что популяция мономорфна относительно аллеля $a, 2$ - популяция мономорфна относительно аллеля $A$

3) колебания генетического состава:

$$
(\bar{q}=0, \bar{p}=1) \text { при } q_{0}<p^{*}, p_{0}>p^{*},(\bar{q}=1, \bar{p}=0) \text { при } q_{0}>p^{*}, p_{0}<p^{*},
$$

где $p^{*}=\frac{r_{a a}-r_{A a}}{r_{A A}+r_{a a}-2 r_{A a}}$. В частности, если значения репродуктивных потенциалов гомозигот равны, то единичный квадрат делится на 4 равных части, что и видно на рис. $11, a$. Отметим, что $p^{*}$ соответствует равновесному значению частоты аллеля $A$, которое неустойчиво при пониженном репродуктивном потенциале гетерозиготы. Следовательно, если $q_{0}=p^{*}\left(p_{0}=p^{*}\right)$, а $p_{0}<p^{*}\left(q_{0}<p^{*}\right)$, то реализуется неустойчивое решение (16); если же $p_{0}>p^{*}\left(q_{0}>p^{*}\right)$, тогда система попадает в неустойчивое равновесие (17). В случае когда $q_{0}=p^{*}$ и $p_{0}=p^{*}$, несмотря на пониженный репродуктивный потенциал гетерозиготы, будет реализовываться решение (9).

Рассмотрим взаимосвязь генетического состава популяции с ее динамикой (рис. 12). Бассейны притяжения, приведенные на рис. 12, a, демонстрируют, что при одних и тех же значениях демографических параметров в зависимости от текущего генетического состава существует три пути эволюционного развития популяции (рис. 12, a), в рамках каждого из которых динамика численности популяции определяется значением среднего репродуктивного потенциала и процессами саморегуляции (рис. 12, б). Так, если закрепится аллель $a$ и генотип $a a$ вытеснит генотип $A A$, то мономорфная популяция будет демонстрировать нерегулярные колебания в силу высокого репродуктивного потенциала особей $\left(r_{a a}>r_{A A}\right)$ и плотностной регуляции рождаемости (рис. $12, \partial)$. При других условиях может сложиться ситуация, что более перспективная форма не способна естественным образом вытеснить явно более слабого по репродуктивным показателям генетического конкурента, и тогда будет наблюдаться стабилизация численности (рис. $12, e$ ). Однако возможна ситуация, когда будет реализован полиморфизм в виде 2-цикла, в рамках которого генетический состав будет совершать колебания подобные «биениям» и оба генотипа будут оказывать влияние на развитие популяции, в результате чего динамика численности популяции также перейдет к колебаниям (рис. $12,6, ж$ ). При этом изменение текущей численности может привести к смене наблюдаемого режима динамики, если наблюдается мультистабильность, однако направление эволюции сохранится, если соотношение генотипов не изменится (рис. 12, в, г).

Следует отметить, что выявленные закономерности возникновения и эволюции 2-циклов модели (6) и характер динамического поведения популяции при пониженной приспособленности гетерозиготы в большой степени определяются особенностями жизненного цикла особей, составляющих моделируемую популяцию. Именно жизненный цикл определяет наличие субпопуляций четных и нечетных лет, которые фактически являются генетически изолированными, что, в свою очередь, приводит к возможности независимой микроэволюции этих субпопуляций и возникновению сложных сценариев динамики как численности, так и генетической 
(a) Динамика генетического состава

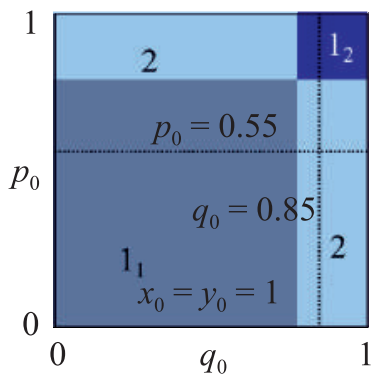

(д)

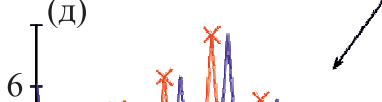

$p_{0}$

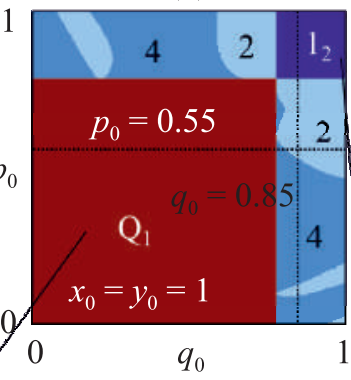

(e)

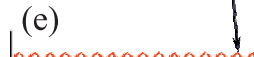

$p_{0}$

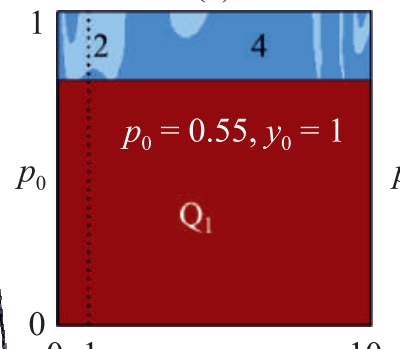

0
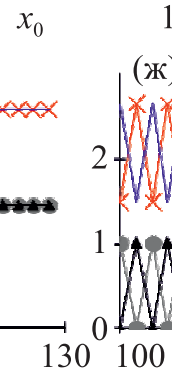

ческого разнообразия

(г)

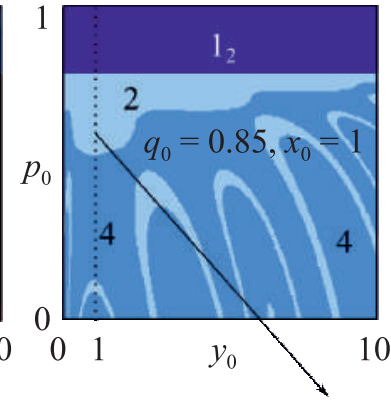

ж)
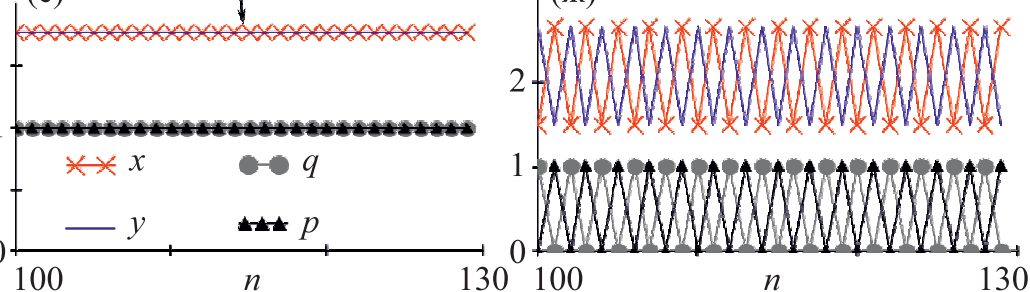

Рис. 12. а-г) Бассейны притяжения динамических режимов модели (6) при $r_{A a}=5, r_{A A}=9.9, r_{a a}=22.4$, $\rho=0.3$. Числа соответствуют длине наблюдаемого цикла. Индекс 1 отражает, что популяция мономорфна относительно аллеля $a, 2$ - популяция мономорфна относительно аллеля $A$. д-ж) Динамика генетического состава и численностей возрастных групп популяции

структуры. Закрепление разных адаптивных мутаций постепенно приведет к различиям в средних репродуктивных потенциалах субпопуляций и достижению ими разного равновесного уровня численности. Дальнейший эволюционный рост репродуктивных потенциалов экологически лимитированных субпопуляций приводит к колебаниям их численностей, которые могут отличаться не только амплитудой, но и фазой.

При повышенном репродуктивном потенциале гетерозиготы (как, впрочем, и при нейтральных мутациях) субпопуляции окажутся полиморфными, но имеющими несколько различающихся по наборам аллелей полиморфных локусов. При пониженной приспособленности гетерозиготы подобные различия труднодостижимы из-за ловушки бистабильности, но, раз установившись, они оказываются весьма устойчивыми, вплоть до выраженной генетической, да и морфологической генетической дифференциации субпопуляций смежных лет.

Наличие двухгодичных колебаний генетического состава и численности субпопуляций смежных лет давно подтверждено разнообразными результатами наблюдений за динамикой популяций тихоокеанской горбуши (Oncorhynchus gorbuscha). В одной реке размножаются особи четных и нечетных генераций этого вида. В результате две субпопуляции четных и нечетных лет порождают двухлетний цикл. Обычно одно поколение многочисленное, другое малочисленное, при этом численность четных и нечетных поколений в одной реке может различаться в десятки раз [Пустовойт, 2011]. Более того, на основе данных об аллозимной изменчивости было показано наличие заметных генетических различий между поколениями четных и нечетных лет [Животовский и др., 1989; Zhivotovsky et al., 1994]. Есть работы, в которых показана и морфологическая дифференциация этих поколений, включая различия по размерно-весовым характеристикам. Все это хорошо укладывается в рамки сценариев эволюции динамических режимов, получаемых в предложенной модели, подтверждая ее адекватность и актуальность.

Заметим, что, по различным грубым оценкам, основанным на данных о динамике локальных популяций и популяционных систем, репродуктивный потенциал популяций горбуши колеблется в пределах от 7 до 14 [Ефанов, 2005]. Для получения достоверной оценки параметра $\rho$ у нас не достаточно данных. Однако, как следует из проведенного анализа, при таких значениях репродуктивного потенциала и малых $\rho$ численность популяции находится в области квазипериодических колебаний, при больших $\rho-$ стабилизируется, а при еще больших - «сваливает- 
ся〉 в режим регулярных колебаний с локальными минимумами в четные годы и максимумами в нечетные (или наоборот). Фактически весь этот спектр динамического поведения численности и описан при наблюдениях за разными популяциями горбуши на Сахалине, Курильских о-вах и Камчатке. Различие в значениях $\rho$ для разных популяций связано, по-видимому, с особенностями распределения ресурсов при нагуле в море и, соответственно, с разным характером конкурентного взаимодействия субпопуляций смежных лет.

Что же касается первичной генетической дивергенции популяций горбуши, то тут ведущую роль играют два обстоятельства. Во-первых, это выраженная пространственная подразделенность и существенная изолированность различных локальных популяций горбуши, вызванная homing-эффектом: возвращением к локальным местам нереста; во-вторых - наличие практически для каждой локальной популяции полностью изолированных субпопуляций смежных лет нереста. Пространственная подразделенность ведет к формированию генетически обособленных пространственных группировок, а наличие изолированных субпопуляций смежных лет нереста обеспечивает независимую микроэволюцию этих субпопуляций и накопление в них различных аллелей как нейтральных, так и адаптивных, приводящих к сосуществованию субпопуляций, обладающих разными репродуктивными потенциалами. В случае движущего отбора, когда гетерозигота имеет промежуточный репродуктивный потенциал, и при этом в одной из субпопуляций фиксирован один аллель, а в другой - другой, такая ситуация потенциально неустойчива. Попадание лучшего аллеля (обеспечивающего больший репродуктивный потенциал гомозиготы) в субпопуляцию, не имеющую ранее этого аллеля, приводит к его фиксации и выравниванию репродуктивных потенциалов субпопуляций (и режимов их динамики). Однако такое «попадание» оказывается невозможно ввиду полной изоляции субпопуляций. И неустойчивое состояние при различии репродуктивных потенциалов субпопуляций, вызванном движущим отбором в одной из них, оказывается фактически устойчивым в силу изолированности субпопуляций и малой вероятности возникновения в них одинаковых мутаций.

Еще более интересное возникновение генетических различий в субпопуляциях связано с мутациями, у которых проявляется пониженная приспособленность гетерозиготы. В этом случае появление генетических различий в субпопуляциях, приводящих к различию их репродуктивных потенциалов и различиям динамики поколений четных и нечетных лет, требует дополнительных условий, обеспечивающих сравнительно большую начальную концентрацию разных аллелей в субпопуляциях, но если такие условия возникнут, то все эти различия окажутся постоянными и устойчивыми.

Что касается оценок репродуктивных потенциалов конкретных генотипов, то такую задачу, по-видимому, изначально не имеет смысла ставить в силу ее бесперспективности. Еще никому не удавалось адекватно оценить приспособленности или репродуктивные потенциалы каких-либо генотипов из природных популяций, тем более для генов-маркеров, оцененных в ходе аллозимного анализа. Грубые оценки возможны для репродуктивных потенциалов субпопуляций, т. е. средних по генотипам, а все остальное - только на качественном уровне. Рассмотренная модель дает такое же разнообразие динамических режимов, которое наблюдается в природных популяциях горбуши, она описывает и объясняет наблюдаемые различия в динамике и генетической структуре субпопуляций смежных лет. Этого вполне достаточно, чтобы признать модель полезной и адекватной.

Все дальнейшие модельные исследования популяций горбуши следует, по-видимому, сосредоточить вокруг моделирования пространственных группировок популяций, связанных миграциями, т. е. заниматься моделированием пространственно-временной динамики метапопуляций. Предлагаемые здесь модели окажутся элементарными компонентами больших систем. Основной упор можно сделать на оценку пространственных корреляций как динамических режимов, так и генетических структур локальных популяций и субпопуляций смежных поколений. Основная трудность - фрагментарность и непоследовательность полевых исследований и наблюдений. Даже данные об объемах официального промысла крайне трудно найти и проверить. Однако качественные интересные результаты получить можно, и поле деятельности здесь необъятное и захватывающее. 


\section{Заключение}

В работе на примере простой модельной ситуации, когда все адаптивное разнообразие определяется одним аутосомным диаллельным локусом, исследована эволюция режимов динамики численности лимитированной популяции биологического вида с неперекрывающимися поколениями и стадийным развитием особей: за время жизненного цикла они проходят две стадии, возможно в различающихся средах обитания.

В ходе исследования показано, что генетический состав популяции (а именно, будет ли она полиморфной или мономорфной) определяется значениями репродуктивных потенциалов гетерозиготы и гомозигот. При этом режимы динамики численности популяции определяются величиной среднего репродуктивного потенциала зрелых особей и интенсивностью процессов саморегуляции. В частности, показано, что эволюционный рост среднего значения репродуктивного потенциала при плотностной регуляции рождаемости приводит к дестабилизации динамики численности возрастных групп, в то время как интенсивность процессов саморегуляции определяет характер возникающих колебаний, поскольку от соотношения степени воздействия численностей разных возрастных групп на снижение рождаемости зависит сценарий потери устойчивости равновесных состояний.

Показано, что закономерности возникновения и эволюции циклических режимов динамики в большой степени определяются особенностями жизненного цикла особей, составляющих популяцию. Именно жизненный цикл определяет наличие изолированных субпопуляций разных лет, что, в свою очередь, приводит к возможности независимой микроэволюции этих субпопуляций и возникновения сложных сценариев динамики как численности, так и генетической структуры. Закрепление разных адаптивных мутаций постепенно приведет к генетической (а возможно, и морфологической) дифференциации и к различиям в средних репродуктивных потенциалах субпопуляций и достижению ими разного равновесного уровня численности. Дальнейший эволюционный рост репродуктивного потенциала экологически лимитированных субпопуляций приводит к колебаниям их численности, которые могут отличаться не только амплитудой, но и фазой.

Обнаруженные в предложенной модели сценарии микроэволюции генетического состава популяции, связанные с колебаниями численности, вполне согласуются с результатами исследования популяции тихоокеанской горбуши, которая демонстрирует не только колебания численности, но и наличие генетически дифференцированных субпопуляций смежных поколений.

\section{Список литературы (References)}

Гиммельфарб А. А., Гинзбург Л. Р., Полуэктов Р. А., Пьхх Ю. А., Ратнер В. А. Динамическая теория биологических популяций. - М.: Наука, 1974.

Gimmel'farb A. A., Ginzburg L. R., Poluektov R. A., Py'kh Yu. A., Ratner V. A. Dinamicheskaya teoriya biologicheskikh populyacij [The dynamic theory of biological populations]. - Moscow: Nauka, 1974 (in Russian).

Дажо Р. Основы экологии. - М.: Прогресс, 1975.

Dajor R. Precis d'ecologie. - Paris, 1972. (Russ. ed.: Dazho R. Osnovy ekologii. — Moscow: Progress, 1975.)

Eфремов В. В. Аллозимная изменчивость горбуши Oncorhynchus gorbuscha Сахалина // Вопр. ихтиол. - 2002. - Т. 42, № 3. - С. 409-417.

Efremov V. V. Allozyme Variation in Pink Salmon Oncorhynchus gorbuscha from Sakhalin Island // Journal of Ichthyology. - 2002. - Vol. 42, No. 4. - P. 339-347. (Original Russian paper: Efremov V. V. Allozimnaya izmenchivost' gorbushi Oncorhynchus gorbuscha Sakhalina // Vopr. ikhtiol. — 2002. - Vol. 42, No. 3. - P. 409-417.)

Ефанов В. Н. Организация мониторинга и моделирование запасов рыб на примере горбуши: диссертация на соискание ученой степени доктора биологических наук. - СПб.: СПбГУ, 2005 .

Efanov $V$. N. Organizatsiya monitoringa i modelirovanie zapasov ryb na primere gorbushi [Organization of monitoring and modeling of fish stocks on the example of pink salmon]: dissertatsiya na soiskanie uchenoi stepeni doktora biologicheskikh nauk [dissertation for the degree of Doctor of Biological Sciences]. — St. Petersburg: SPbGU, 2005. 
Жданова О. Л., Фрисман Е. Я. Нелинейная динамика численности популяции: влияние усложнения возрастной структуры на сценарии перехода к хаосу // Журнал общей биологии. 2011. - T. 72, № 3. - C. 214-229.

Zhdanova O. L., Frisman E. Ya. Nonlinear population dynamics: Complication of the age structure influences transition to chaos scenarios // Biology Bulletin Reviews. - 2011. - Vol. 1, No. 5. - P. 395-406. (Original Russian paper: Zhdanova O. L., Frisman E. Ya. Nelineynaya dinamika chislennosti populyatsii: vliyaniye uslozhneniya vozrastnoy struktury na stsenarii perekhoda $\mathrm{k}$ khaosu // Zhurnal obshchey biologii. — 2011. — Vol. 72, No. 3. - P. 214-229.)

Животовский Л. А., Глубоковский М. К., Викторовский Р. М., Броневский А. М., Афанасьев К. И., Ефремов В. В., Ермоленко Л. Н., Калабушкин Б. А., Ковалев В. Г., Макоедов А. Н., Малинина Т. В., Пустовойт С. П., Рубичова Г. А. Генетическая дифференциация горбуши // Генетика. - 1989. - Т. 25, № 7. - С. 1261-1274.

Zhivotovskij L. A., Glubokovskij M. K., Viktorovskij R. M., Bronevskij A. M., Afanas'ev K. I., Efremov V. V., Ermolenko L. N., Kalabushkin B. A., Kovalev V. G., Makoedov A. N., Malinina T. V., Pustovoit S. P., Rubtsova G. A. Geneticheskaya differenciaciya gorbushi [Genetic differentiation of pink salmon] // Genetika. — 1989. — Vol. 25, No. 7. P. 1261-1274 (in Russian).

Кузнеиов А. П., Кузнецов С. П., Поздняков М. В., Седова Ю. В. Универсальное двумерное отображение и его радиофизическая реализация // Нелинейная динамика. - 2012б. - Т. 8, № 3. - С. 461-471.

Kuzneczov A. P., Kuzneczov S. P., Pozdnyakov M. V., Sedova Yu.V. Universal'noe dvumernoe otobrazhenie i ego radiofizicheskaya realizaciya [Universal two-dimensional map and its radiophysical realization] // Rus. J. Nonlin. Dyn. - 2012. - Vol. 8, No. 3. - P. 461-471 (in Russian).

Кузнецов А. П., Савин А. В., Седова Ю. В., Тюрюкина Л. В. Бифуркации отображений. - Саратов: Наука, 2012а.

Kuzneczov A. P., Savin A. V., Sedova Yu. V., Tyuryukina L. V. Bifurkacii otobrazhenij [Bifurcations of mappings]. Saratov: Nauka, 2012 (in Russian).

Неверова Г. П., Жданова О.Л., Фрисман Е. Я. Возникновение сложных режимов динамики численности в ходе эволюции структурированной лимитированной популяции // Генетика. - 2020. — T. 56, № 6. - С. 714-725. — DOI: 10.31857/S0016675820060065

Neverova G. P., Zhdanova O. L., Frisman E. Ya. The Emergence of Complex Dynamics during the Evolution of a Structured Limited Population // Russian Journal of Genetics. — 2020. — Vol. 56, No. 6. — P. 747-757. — DOI: 10.1134/S102279542006006X. (Original Russian paper: Neverova G. P., Zhdanova O. L., Frisman E. Ya. Vozniknovenie slozhnykh rezhimov dinamiki chislennosti $\mathrm{v}$ khode evolyucii strukturirovannoj limitirovannoj populyacii // Genetika. - 2020. - Vol. 56, No. 6. - P. 714-725.)

Неверова Г. П., Фрисман Е.Я. Сравнительный анализ влияния различных типов плотностной регуляции на динамику численности структурированных популяций // Информатика и системы управления. — 2015. - № 1 (43). - С. 41-53.

Neverova G. P., Frisman E. Ya. Sravnitel'nyy analiz vliyaniya razlichnykh tipov plotnostnoy regulyatsii na dinamiku chislennosti strukturirovannykh populyatsiy [Comparative analysis of the influence of various types of density regulation on the dynamics of the number of structured populations] // Information Science and Control Systems. - 2015. Vol. 43, No. 1. - P. 41-53 (in Russian).

Новиков Е. А., Панов В. В., Мошкин М. П. Плотностно-зависимые механизмы регуляции численности красной полевки (Myodes rutilus) в оптимальных и суботимальных местообитаниях юга Западной Сибири // Журн. общей биол. - 2012. - Т. 73, № 1. - С. 49-58.

Novikov E. A., Panov V. V., Moshkin M. P. Density-dependent regulation in populations of northern red-backed voles (Myodes rutilus) in optimal and suboptimal habitats of southwest Siberia // Biology Bulletin Reviews. - 2012. Vol. 2, No. 5. - P. 431-438. (Original Russian paper: Novikov E. A., Panov V. V., Moshkin M. P. Plotnostno-zavisimye mexanizmy regulyacii chislennosti krasnoj polevki (Myodes rutilus) v optimal'nykh i subotimal'nykh mestoobitaniyax yuga Zapadnoj Sibiri // Zhurn. obshhej biol. — 2012. — Vol. 73, No. 1. — P. 49-58.)

Одум Ю. Основы экологии. - М.: Мир, 1975.

Odum E. P. Fundamentals of Ecology: 3d Ed. — Saunders, 1971. (Russ. ed.: Odum Yu. Osnovy ekologii. — Moscow: Mir, 1975.)

Пустовойт С. П. Основные итоги генетического мониторинга североохотоморских популяций горбуши (Oncorhynchus gorbuscha) // Вавилов. журн. генетики и селекции. - 2011. T. 15, № 3. - C. 475-484.

Pustovojt S. P. Osnovnye itogi geneticheskogo monitoringa severookhotomorskikh populyacij gorbushi (Oncorhynchus gorbuscha) [Principal results of genetic monitoring of pink salmon (Oncorhynchus gorbuscha) populations from 
the northern coast of the sea of Okhotsk] // Vavilov journal of genetics and breeding. — 2011. — Vol. 15, No. 3. — P. 475-484 (in Russian).

Пустовойт С. П. Тридцать лет гипотезе флюктуирующих стад горбуши Oncorhynchus gorbuscha (Walbaum) // Известия ТИНРО. - 2017. - Т. 188. - С. 162-172.

Pustovoit S. P. Tridczat' let gipoteze flyuktuiruyushhikh stad gorbushi Oncorhynchus gorbuscha (Walbaum) [Thirty years of the hypothesis on fluctuating stocks of pink salmon Oncorhynchus gorbuscha (Walbaum)] // Izv. TINRO. 2017. - Vol. 188. - P. 162-172 (in Russian).

Свирежев Ю. М., Логофет Д. О. Устойчивость биологических сообществ. - М.: Наука, 1978. Svirezhev Yu. M., Logofet D. O. Ustoychivost' biologicheskikh soobshchestv [Stability of biological communities]. Moscow: Nauka, 1978 (in Russian).

Уильямсон М. Анализ биологических популяций / пер. с англ. А. Д. Базыкина. - М.: Мир, 1975.

Wiliamson M. The analysis of biological population. - London, 1972. (Russ. ed.: Uil'yamson M. Analiz biologicheskikh populyacij / per. s angl. A. D. Bazykina. - Moscow: Mir, 1975.)

Фрисман Е. Я., Жданова О. Л. Эволюционный переход к сложным режимам динамики численности двухвозрастной популяции // Генетика. — 2009. — Т. 45, № 9. - С. 1277-1286.

Frisman E. Ya., Zhdanova O.L. Evolutionary transition to complex population dynamic patterns in a two-age population // Rus. J. Genet. — 2009. — Vol. 45, No. 9. - P. 1224-1133. (Original Russian paper: Frisman E. Ya., Zhdanova $O$. L. E'volyucionny ’j perexod k slozhny`m rezhimam dinamiki chislennosti dvuxvozrastnoj populyacii // Genetika. - 2009. - Vol. 45, No. 9. - P. 1277-1286).

Фрисман Е.Я. Странные аттракторы в простейших моделях динамики численности популяций с возрастной структурой // Доклады академии наук. - 1994. - Т. 338, № 2. C. 282-286.

Frisman E. Ya. Strannyye attraktory v prosteyshikh modelyakh dinamiki chislennosti populyatsiy s vozrastnoy strukturoy [Strange attractors in the simplest models of population dynamics with age structure] // Doklady akademii nauk. — 1994. - Vol. 338, No. 2. - P. 282-286 (in Russian).

Фрисман Е. Я., Неверова Г. П., Кулаков М. П., Жигальский О. А. Смена динамических режимов в популяциях видов с коротким жизненным циклом: результаты аналитического и численного исследования // Математическая биология и биоинформатика. - 2014. - Т. 9, № 2. - C. 414-429.

Frisman E. Ya., Neverova G. P., Kulakov M. P., Zhigalskii O. A. Smena dinamicheskikh rezhimov v populyatsiyakh vidov s korotkim zhiznennym tsiklom: rezul'taty analiticheskogo i chislennogo issledovaniya [Changing the dynamic modes in populations with short life cycle: mathematical modeling and simulation] // Mathematical Biology and Bioinformatics. - 2014. - Vol. 9, No. 2. — P. 414-429 (in Russian).

Фрисман Е. Я., Кулаков М. П., Ревуикая О. Л., Жданова О. Л., Неверова Г. П. Основные направления и обзор современного состояния исследований динамики структурированных и взаимодействующих популяций // Компьютерные исследования и моделирование. 2019. — T. 11, № 1. - C. 119-151. — DOI: 10.20537/2076-7633-2019-11-1-119-151

Frisman E.Ya., Kulakov M.P., Revutskaya O.L., Zhdanova O.L.,Neverova G.P. Osnovny`e napravleniya i obzor sovremennogo sostoyaniya issledovanij dinamiki strukturirovanny`x i vzaimodejstvuyushhix populyacij [Dynamics of structured and interacting populations] // Computer Research and Modeling. — 2019. - Vol. 11, No 1. - P. 119-151. DOI: 10.20537/2076-7633-2019-11-1-119-151 (in Russian).

Фрисман Е. Я., Ласт Е. В., Лазуткин А. Н. Механизмы и особенности сезонной и долговременной динамики популяций полевок Clethrionomys rufocanus и Cl. rutilus: количественный анализ и математическое моделирование // Вестн. Сев.-Вост. науч. центра Дальневост. отд. PAH. - 2010. — № 2. - C. 43-47.

Frisman E. Ya., Last E. V., Lazutkin A. N. Mekhanizmy i osobennosti sezonnoy i dolgovremennoy dinamiki populyatsiy polevok Clethrionomys rufocanus i Cl. rutilus: kolichestvennyy analiz i matematicheskoye modelirovaniye [The Mechanisms and Peculiar Characters of Seasonal and Long-Term Dynamics of Voles Clethrionomys rufocanus and Cl. rutilus: a Quantitative Study and Mathematical Modeling] // Bulletin of the North-East Scientific Center, Russia Academy of Sciences Far East Branch. - 2010. — No. 2. - P. $43-47$ (in Russian).

Чернявский Ф. Б., Лазуткин А. Н. Циклы леммингов и полевок на Севере. - Магадан: ИБПС ДВО РАН, 2004.

Chernyavskij F. B., Lazutkin A. N. Cikly lemmingov i polevok na Severe [Cycles of lemmings and voles in the North]. - Magadan: IBPS DVO RAN, 2004 (in Russian). 
Шапиро А. П. Роль плотностной регуляции в возникновении колебаний численности многовозрастной популяции // Исследования по математической популяционной экологии. — Владивосток: ДВНЦ АН СССР, 1983. - С. 3-17.

Shapiro A. P. Rol' plotnostnoj regulyacii v vozniknovenii kolebanij chislennosti mnogovozrast-noj populyacii [The role of density regulation in the occurrence of fluctuations in the abundance of a multi-aged population] // Issledovaniya po matematicheskoj populyacionnoj ehkologii [Studies in mathematical population ecology]. — Vladivostok: DVNC AN SSSR. 1983. - P. 3-17 (in Russian).

Шапиро А. П., Луппов С. П. Рекуррентные уравнения в теории популяционной биологии. — М.: Наука, 1983.

Shapiro A. P., Luppov S. P. Rekurrentnye uravneniya v teorii populyacionnoj biologii [Recurrent equations in the theory of population biology]. - Moscow: Nauka, 1983 (in Russian).

Barraquand F., Louca S., Abbott K. C., Cobbold C. A., Cordoleani F., DeAngelis D. L., Elderd B. D., Fox J. W., Greenwood P., Hilker F. M., Murray D. L., Stieha C. R., Taylor R. A., Vitense K., Wolkowicz G. S. K., Tyson R. C. Moving forward in circles: challenges and opportunities in modelling population cycles // Ecology letters. — 2017. — Vol. 20, No. 8. - P. 1074-1092.

Beacham T. D., McIntosh B., MacConnachie C., Spilsted B., White B. A. Population structure of pink salmon (Oncorhynchus gorbuscha) in British Columbia and Washington, determined with microsatellites // Fishery Bulletin. - 2012. - Vol. 110, No. 2. - P. 242-256.

Boer P. J., Reddingius J. Regulation and Stabilization Paradigms in Population Ecology. - Netherlands: Chapman \& Hall Ltd, 1996.

Frisman E., Neverova G., Revutskaya O. Complex Dynamics of the Population with a Simple Age Structure // Ecological Modelling. — 2011. - Vol. 222. — P. 1943-1950.

Ginzburg L., Colyvan M. Ecological Orbits: How Planets Move And Populations Grow. - New York: Oxford University Press, 2004.

Gurney W., Nisbet R. Ecological Dynamics. - New York: Oxford University Press, 1998.

Inchausti P., Ginzburg L. Small mammals cycles in northern Europe: patterns and evidence for the maternal effect hypothesis // J. of Animal Ecology. - 1998. — Vol. 67. — P. 180-194

Krebs C. J. Population Fluctuations in Rodents. - Chicago: The University of Chicago Press, 2013.

Lack D. The Natural Regulation of Animal Numbers. — New York: Oxford University Press, 1954.

May R. M. Biological populations with non-overlapping generations: stable points, stable cycles and chaos // Science. - 1974. - Vol. 186. - P. 645-647.

Neverova G. P., Abakumov A. I., Yarovenko I. P., Frisman E. Ya. Mode change in thedynamics of exploited limited population with age structure // Nonlinear Dynamics. - 2018. - Vol. 94. P. 827-844. - DOI: 10.1007/s11071-018-4396-6

Ricker W. E. Stock and recruitment // J. Fish. Res. Board Can. - 1954. — Vol. 5, No. 5. - P. 559-623.

Sato S., Urawa S. Genetic variation of Japanese pink salmon populations inferred from nucleotide sequence analysis of the mitochondrial DNA control region // Environmental biology of fishes. 2017. - Vol. 100, No. 10. - P. 1355-1372.

Zhivotovsky L. A., Gharret A. J., McGregor A. J., Glubokovsky M. K., Feldman M. W. Gene differentiation in pacific salmon (Oncorhynchus sp.): facts and models with reference to pink salmon (O. gorbuscha) // Can. J. Fish. and Aquat. Sci. — 1994. — Vol. 51 (S1). - P. 223-232. 